\title{
ESTIMATION FOR BILINEAR STOCHASTIC SYSTEMS
}

Alan S. Willsky.

Steven I. Marcus

Electronic Systems Laboratory 


\title{
ESTIMATION FOR BILINEAR
}

STOCHASTIC SYSTEMST

Alan S. Willsky* and Steven I. Marcus**

\author{
Electronic Systems Laboratory \\ Department of Electrical Engineering \\ Massachusetts Institute of rechnology \\ Cambridge, Massachusetts 02139
}

\section{ABSTRACT}

In this paper we consider several applications of bilinear stochastic models in which state estimation is an important problem. Bilinear stochastic models occur naturally in many communication problems, including noisy oscillators and phase-lock loops, in which the system evolves on the circle $\mathrm{s}^{1}$. Similar models arise in the estimation of the position of an orbiting body (in which the state evolves on the 2-sphere $\mathrm{S}^{2}$ ) and in the estimation of the orientation of a rotating rigid body (which evolves on so(3)). The advection-diffusion model of air pollution involves partial differential equations which, when discretized, include bilinear stochastic terms due to the random fluctuations in wind velocity and source rate.

Three techniques for the solution of bilinear estimation problems are presented. First, finite dimensional optimal nonlinear estimators are presented for certain bilinear systems evolving on solvable and nilpotent Lie groups. Then the use of harmonic analysis for estimation problems evolving on spheres and other compact manifolds is investigated. Finally, an approximate estimation technique utilizing cumulants is discussed.

TThis paper is to be presented at the U.S. - Italy Seminar on Variable Structure Systems, Oregon, May 26-31, 1974.

*The work of this author was supported in part by NASA under Grant NGL-22-009-124 and in part by NSF under Grant GK-42090.

\section{**National Science Foundation Fellow}




\section{Introduction}

As is well known, the class of linear dynamical systems with white gaussian driving and observation noises is particularly appealing in that optimal estimation and control systems can be readily determined and are easily implemented (perhaps with the aid of a digital computer). Unfortunately, there exists no such "nice" theory for general finitedimensional nonlinear systems, and until recently most nonlinear estimation problems were "solved" by various types of linearization and vector space approximation methods.

Recently, a great deal of effort has gone into studying a class of nonlinear systems that possesses a great deal of structure itself -the class of bilinear systems. Several authors have been able to devise analytical techniques for such systems that are as detailed and as powerful as those for linear systems. Moreover, the mathematical tools behind bilinear system analysis include not only many of the vector space techniques that are so valuable in linear system theory but also a number of tools drawn from the theories of Lie groups and differential geometry. This points out the necessity of viewing the dynamical system of interest in its most natural setting, rather than forcing it into the vector space framework.

Both the Iie theoretic and vector space settings have proven to be useful in the study of bilinear estimation problems, and a number of important and illuminating results have been uncovered. It is the purpose of this paper to explain the practical and mathematical importance of these results. In section II we view the basic mathematical formulation 
of interest to us, and in Sections III-V we discuss several important practical problems that fall within this framework. These include a large class of synchronous communication problems, the problems of altitude estimation and the tracking of an orbiting vehicle, and the estimation of pollutant concentrations in a diffusive atmosphere subject to random wind effects and fluctuations in the source rate. These by no means cover all of the potential applications for our bilinear estimation results, but they do indicate the range of problems that can be considered. We refer the reader to the references for other applications. In Sections VI-VIII we review the techniques that have been developed for bilinear estimation. In section VI we describe a class of bilinear systems for which complete analysis is possible, and we display the optimal, nonlinear, finite-dimensional estimation equations for an example. The Lie-theoretic significance of these results is also discussed. In Section VII the use in estimation system design of harmonic analysis on groups is explored in the context of synchronous communication and orbital tracking. This represents a potentially powerful tool in the design of high performance, implementable estimation systems. A second approximation method, based on the truncation of the cumulants of a random process, is studied in Section VIII. This approach is more closely related to the usual vector space techniques. 


\section{Stochastic Bilinear systems}

In this section we briefly describe the several ciasses of stochastic equations that will be considered in the remaining sections of this paper. The basic deterministic bilinear equation considered in the literature [1] - [9] is

$$
\dot{x}(t)=\left[A_{0}+\sum_{i=1}^{N} u_{i}(t) A_{i}\right] x(t)
$$

where the $A_{i}$ are given $n \times n$ matrices, the $u_{i}$ are scalar inputs, and $x$ is either an n-vector or an $n \times n$ matrix. As discussed in [1], the additive control model

$$
\dot{x}(t)=\left[B_{0}+\sum_{i=1}^{N} u_{i}(t) B_{i}\right] x(t)+C u(t)
$$

(here $u$ is the vector of the $u_{i}$ ) can be reduced to the form (2.1) by state augmentation. Also, if we apply the bilinear feedback law

$$
u_{i}(t)=v_{i}(t) \ell_{i}(x(t))+\mu_{i}(t)
$$

where $v_{i}$ and $\mu_{i}$ are scalars, and $\ell_{i}$ is a scalar-valued linear function of $x$, our system equation becomes

$$
\dot{x}(t)=\left[A_{0}+\sum_{i=1}^{N}\left(v_{i}(t) \ell_{i}(x(t))+\mu_{i}(t)\right) A_{i}\right] x(t)
$$

which involves products of state variables. By including several feedback paths, we can obtain essentially arbitrary polynomials in the state variables.

In this paper we will consider equations such as (2.1) in which the $u_{i}$ are.stochastic processes. Such systems have become considered by several authors [9] - [27]. We refer the reader to [10] - [16] for 
detailed discussions of the properties of such stochastic models.

One must be careful in considering stochastic versions of (2.1). For instance, if $u(t)$ is a vector zero mean white noise with

$$
E[u(t) u(s)]=R(t) \delta(t-s)
$$

the Ito stochastic differential analog of (2.1) is

$$
d x(t)=\left\{\left[A_{0}+\frac{1}{2} \sum_{i, j=1}^{N} R_{i j}(t) A_{i} A_{j}\right] d t+\sum_{i=1}^{N} A_{i} d v_{i}(t)\right\} x(t)
$$

where $v$ is the integral of $u$ (i.e., it is a Brownian motion process). We define $I=\left\{A_{i}\right\}_{L A}$ to be the matrix Lie algebra genexated by $\left\{A_{i}\right\}_{i=0}^{N}--i . e .$, it is the smallest subspace that contains the $\left\{A_{i}\right\}$ and is closed under the commutator product

$$
\left[M_{1}, M_{2}\right]=M_{1} M_{2}-M_{2} M_{1}
$$

One can show that in the deterministic case with $x$ an $n \times n$ matrix, if $x(0)$ is an element of the matrix Lie group

$$
G=\{\exp L\}_{G}=\left\{e^{B_{1}} e^{B_{2}} \ldots e^{B_{m}^{m}} \mid B_{i} \varepsilon L\right\}
$$

then $x(t)$ is an element of $G$ (for all $t \geq 0$ ). In order to make a similar statement in the stochastic case when $u$ is a white noise, we must include a correction term, as indicated in (2.6).

Another case of considerable importance arises if $\mathrm{u}$ is generated by a finite dimensional linear diffusion process 


$$
\begin{gathered}
d \xi(t)=F(t) \xi(t) d t+G(t) d w(t)+\alpha(t) d t \\
u(t)=H(t) \xi(t)
\end{gathered}
$$

where $\alpha, F, G$, and $H$ are known and $w$ is a standard Brownian motion process (E $\left.\left[d w(t) d w^{\prime}(t)\right]=I d t\right)$. In this case, $\mathbf{x}$ by itself is not a Markov process, but the pair $(x, \xi)$ is. Augmenting the state with $\xi$, we obtain a stochastic equation of the form (2.4), where the $v_{i}=I$ and some of the $\mu_{i}$ are white noises while the others are zero (see several examples in the following sections). We note that one can show [16], [17] that in this case no correction term need be added to (2.4). Also, the righthand side of (2.4) does not satisfy the global Lipschitz conditions often assumed in proving the existence of solutions to I to differential equations [28], [29]. Again, one can shown [15], [20], [30], [31] that this causes no problems in the case when (2.4) arises from (2.1) driven by the colored noise $(2.9)-(2.10)$.

In the remaining sections of this paper, we will consider the estimation of processes described by stochastic bilinear equations of the types just discussed. We now briefly describe the various types of measurement processes that will be considered. We also refer the reader to the references [16] - [27] for more on these estimation problems.

One very important measurement process consists of linear measurements corrupted by additive noise

$$
d z(t)=L(x(t)) d t+d v(t)
$$

where $\mathrm{I}$ is a linear operator (recall $\mathrm{x}$ is either an $\mathrm{n}$-vector or an $\mathrm{n} x \mathrm{n}$ 


$$
-6-
$$

matrix) and $v$ is a Brownian motion process. A second observation model is the "multiplicative measurement noise" case

$$
Z(t)=X(t) V(t)
$$

in which $\mathrm{z}, \mathrm{x}$ and $\mathrm{v}$ are all $\mathrm{n} \mathrm{x} \mathrm{n}$ matrices. The third and final measurement process is described by a bilinear dynamical equation

$$
\begin{aligned}
d z(t)=\left\{\left[A_{0}\right.\right. & \left.+\frac{1}{2} \sum_{i, j=1}^{N} R_{i j}(t) A_{i} A_{j}\right] d t \\
& \left.+\sum_{i=1}^{N}\left[\ell_{i}(x(t)) d t+d \dot{v}_{i}(t)\right] A_{i}\right\} z(t)
\end{aligned}
$$

Examples of each of these processes will be given in the next few sections. We close this section by noting that many but not all of our results are motivated by considering these estimation problems in the context of Lie group theory. We refer the reader to $[1]-[5],[10],[16]$, [17], and [20] - [23] for more on this subject. 
IIr. Estimation Problems Arising in Communication Applications

An important problem in a large number of communications applications $[10],[17],[19],[24],[25],[32]-[37]$ is the processing of a signal of the form

$$
r(t)=A(t) \sin \left(w_{C} t+\phi(t)+v(t)\right)+N(t)
$$

where $w_{C}$ is a known carrier fxequency, $\phi$ is some type of modulating information, $v$ is a random phase drift, $A$ is the sinusoidal amplitude, possibly containing modulating information and/or noise, and $\mathrm{N}$ is additive channel noise. As discussed in [10], [17], [19], [24], [25], and [34], a number of specific problems that fit into the general form given by (3.1) can be modeled by equations of the type described in the preceding section. In this section we illustrate these ideas by considering several specific examples.

Example 1: We consider a phase tracking problem of importance in radio navigation systems such as Omega [38]. This problem has been studied in [24], [25], [32] - [34] and [36]. The solution technique developed in [24], [25] is discussed in section VII.

$$
\begin{aligned}
& \text { Suppose we receive the signal } \\
& \qquad \dot{z}(t)=\sin \theta(t)+r^{1 / 2}(t) \dot{w}(t)
\end{aligned}
$$

where

$$
\theta(t)=w_{c} t+\int_{0}^{t} q^{1 / 2}(s) d s+\theta_{0}
$$

and $\mathrm{v}$ and $\mathrm{w}$ are independent standard Brownian motions, $\mathrm{q}(\mathrm{t}) \geq 0$, $r(t)>0$, and $w_{c}>0$. Also $\theta_{0}$ is a ranciom initial condition independent of $v$ and $w$. We desire to track the signal phase -- i.e., we wish to

$$
-7-
$$


estimate $\theta(t)$ mod $2 \pi$ given $\{z(s) \mid 0 \leq s \leq t\}$. Equation (3.2) is, of course, only formal, since $w$ is white noise. The I to differential forms of (3.2) and (3.3) are

$$
\begin{aligned}
& d \theta(t)=w_{c} d t+q^{1 / 2}(t) d v(t), \theta(0)=\theta_{0} \\
& d z(t)=\sin \theta(t) d t+r^{1 / 2}(t) d w(t)
\end{aligned}
$$

and we take as our optimal estimation criterion the minimization of

$$
E[(1-\cos (\theta(t)-\tilde{\theta}(t)) \mid z(s), 0 \leq s \leq t] .
$$

Noting that we are essentially tracking a point on the unit circle $\mathbf{S}^{1}$ in the plane $\mathrm{R}^{2}$, we can reformulate our problem in Cartesian coordinates. Let

$$
x_{1}=\sin \theta(t), \quad x_{2}=\cos \theta(t)
$$

then

$$
\begin{gathered}
{\left[\begin{array}{ll}
d x_{1} & (t) \\
d x_{2} & (t)
\end{array}\right]=\left[\begin{array}{ccc}
-q(t) d t / 2 & w_{c} d t+q^{1 / 2} & (t) d v(t) \\
-\left(w_{c} d t+q^{1 / 2}\right. & (t) d v(t)) & -q(t) d t / 2
\end{array}\right]\left[\begin{array}{l}
x_{1}(t) \\
x_{2}(t)
\end{array}\right]} \\
d z(t)=x_{1}(t) d t+x^{1 / 2}(t) d w(t)
\end{gathered}
$$

which are of the bilinear process - linear measurement type discussed in Section II. Note that (3.7) describes what appears to be a damped oscillator; however the damping terms are the correction terms required by Ito calculus, and one can show that 


$$
E\left(x^{2}(t)+x_{2}^{2}(t)\right)=1
$$

(see [16], [17] for further discussion).

In Cartesian coordinates our estimation problem is to choose an estimate $\left(\tilde{x}_{1}(t), \tilde{x}_{2}(t)\right)$ on the unit circle - i.e., such that

$$
\tilde{x}_{1}(t)+\tilde{x}_{2}(t)=1
$$

If we use the least squares criterion

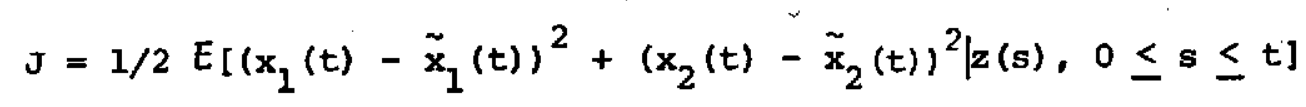

subject to $(3.10)$, or equivalently subject to

$$
\tilde{x}_{1}(t)=\sin \tilde{\theta}(t), \quad \tilde{x}_{2}(t)=\cos \tilde{\theta}(t) .
$$

our criterion reduces to

$$
J=E[1-\cos (\theta(t)-\tilde{\theta}(t)) \mid z(s), 0 \leq s \leq t]
$$

-- i.e. (3.13) represents a contrained least squares criterion. One can show that

$$
\left(\tilde{x}_{1}(t), \tilde{x}_{2}(t)\right)=\frac{\left(\hat{x}_{1}(t \mid t), \hat{x}_{2}(t \mid t)\right)}{\sqrt{\hat{x}_{1}^{2}(t \mid t)+\hat{x}_{2}^{2}(t \mid t)}}
$$

or

$$
\tilde{\theta}(t)=\tan ^{-1} \frac{\hat{x}_{1}(t \mid t)}{\hat{x}_{2}(t \mid t)}
$$

where 


$$
\hat{x}_{i}(t \mid t)=E\left[x_{i}(t) \mid z(s), 0 \leq s \leq t\right], i=1,2
$$

Referring to Figure 3.1 we can see the geometric significance of this criterion. We note that one can show that

$$
P(t)=\sqrt{\hat{x}_{1}^{2}(t \mid t)+\hat{x}_{2}^{2}(t \mid t)} \leq 1
$$

and the quantity $P(t)$ is a measure of our confidence in our estimate. Specifically if $\theta$ is a normal random variable with variance $\gamma$, then (see $[16]-[18],[24],[25])$

$$
P=\sqrt{[E(\sin \theta)]^{2}+[E(\cos \theta)]^{2}}=e^{-\gamma / 2}
$$

so $\gamma=0$ (perfect knowledge of $\theta) \Longrightarrow P=1$

and $\gamma=\infty$ (no knowledge) $\Longrightarrow P=0$.

Example 2: Consider the demodulation of an FM signal in the presence of both phase and additive channel noise. Specifically, suppose the received signal process is

$d z(t)=\sin \left(w_{c} t+g \int_{0}^{t} x(s) d s+\int_{0}^{t} e^{1 / 2}(s) d f(s)\right) d t+x^{1 / 2}(t) d w(t)$

where $x$, the modulating information to be recovered, arises from a linear diffusion process

$$
d x(t)=a(t) x(t) d t+q^{1 / 2}(t) d v(t)
$$

Here $f, w$, and $v$ are independent standard Brownian motion processes. Equations (3.19) and (3.20) can be replaced by equivalanet equations in a manner similar to that used in Example 1. Our state 


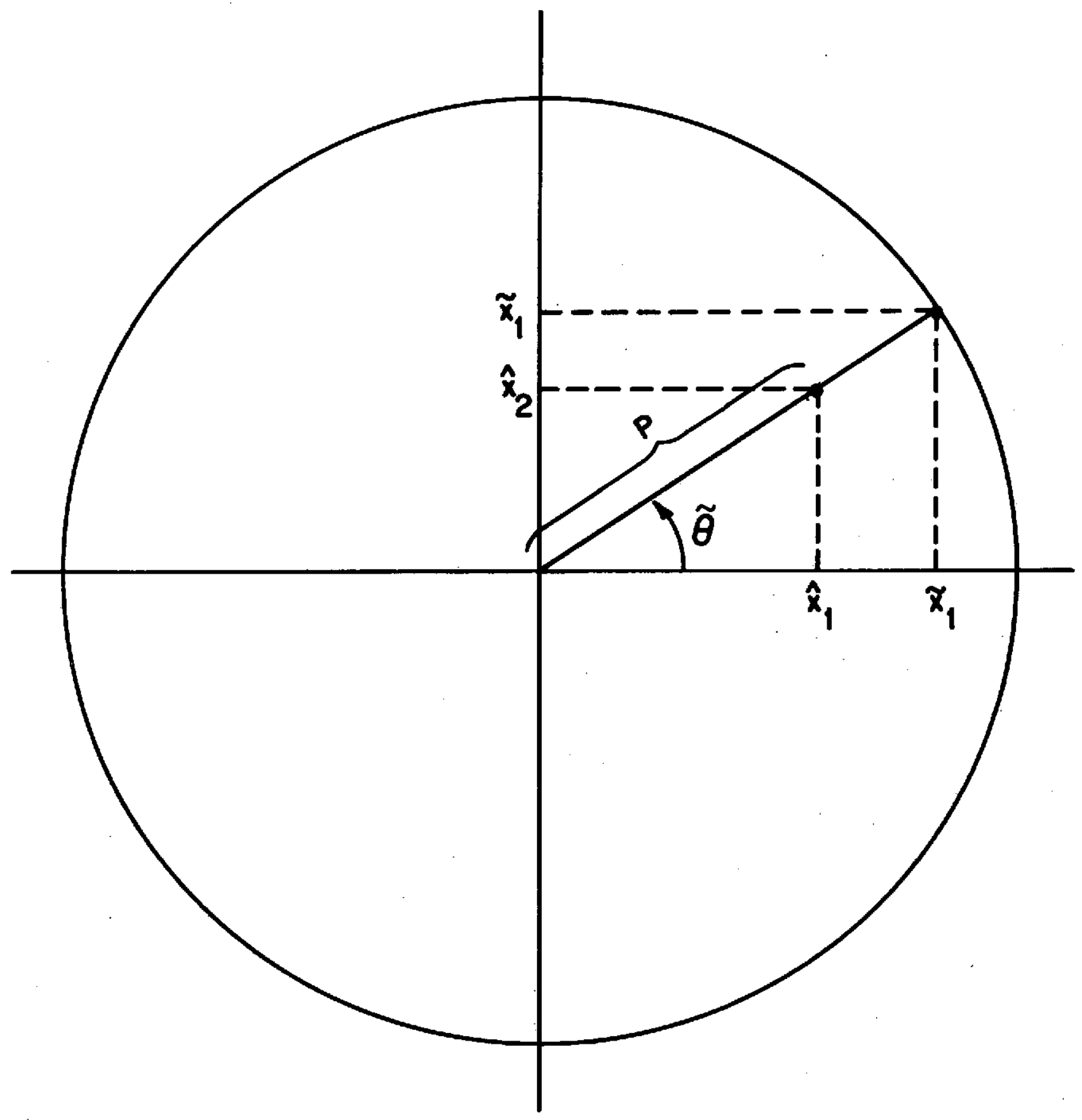

Fig. 3.1 Illustrating the Geometric Interpretation of the Criterion $\varepsilon[1-\cos (\theta-\tilde{\theta})]$ 
equation becomes

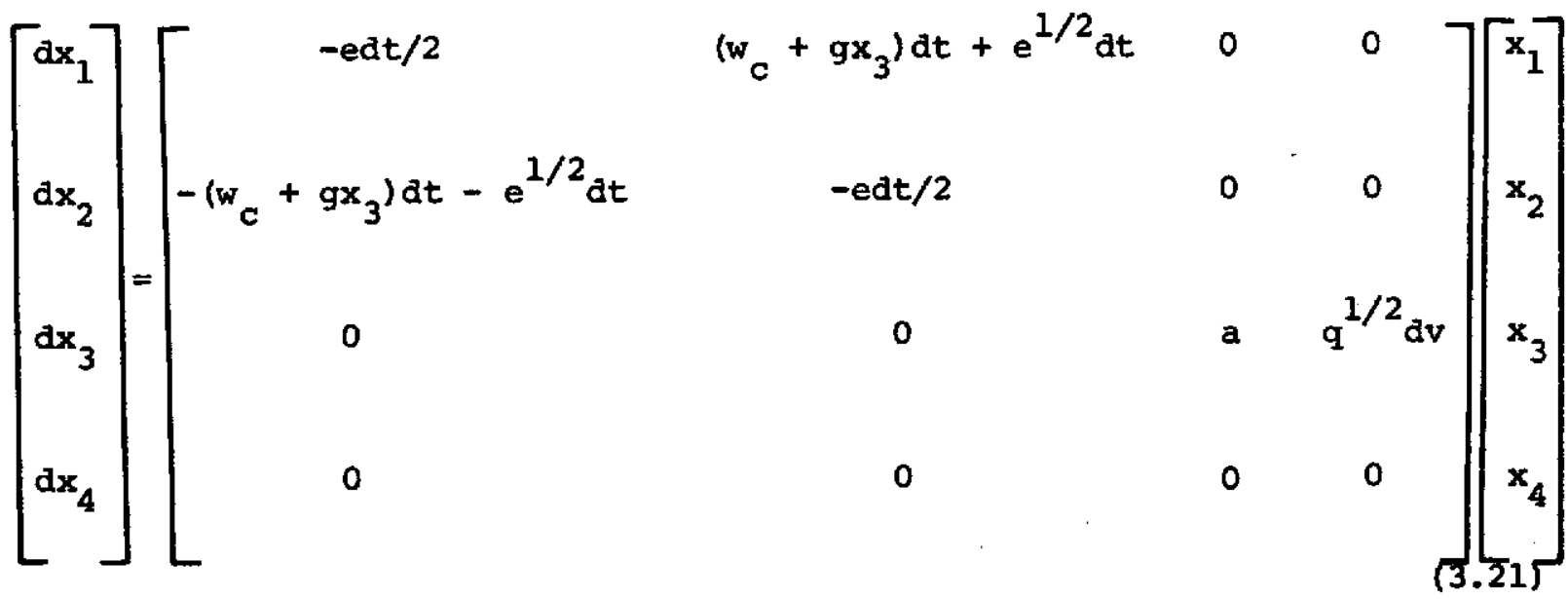

$$
\begin{aligned}
& d z=x_{1} d t+r^{1 / 2} d w(t)
\end{aligned}
$$

where $x_{3}=x$ and $x_{4} \equiv 1$ (this is the type of augmentation we use to "bilinearize" linear systems [1]. Note the products $x_{1} x_{3}$ and $x_{2} x_{3}$ in (3.21).

Example 3: In this example we consider an FM problem with phrase noise only. This problem was considered in [16] - [19], and we refer the reader to [19] for further discussions of examples of this type. Suppose we observe the signal

$$
z_{l}(t)=\sin \left(w_{c} d t+\int_{0}^{t} h(s) x(s) d s+\int_{0}^{t} q^{1 / 2}(s) d w(s)\right)
$$

where $x$ is given by $(3.20)$ and $w$, a standard Brownian motion independent of $x$, represents a random phase drift. A number of physical sources for such noise are discussed in [19]. We note that in standard FM systems involving limiter-discriminators, additive channel noise is processed in such a manner as to yield frequency and phase deviations (see [37], [39]). 
Also, such models arise in the problem of tracking drifts in high quality oscillators used as frequency standards or high-precision clocks [19], [40], in the problem of recovering velocity information from Dopplershifted signals [19], [41].

As discussed in a number of references, given the signal (3.23), there are methods for generating the additional signal

$$
z_{2}(t)=\cos \left(w_{c} t+\int_{0}^{t} h(s) x(s) d s+\int_{0}^{t} q^{1 / 2}(s) d w(s)\right.
$$

(see the method described in [19] in which the total phase of (3.23) is reconstructed with the aid of cycle counters). Defining

$$
\begin{aligned}
& z(t)=\left[\begin{array}{cc}
z_{2}(t) & z_{1}(t) \\
-z_{1}(t) & z_{2}(t)
\end{array}\right] \\
& x(t)=\left[\begin{array}{ll}
\cos \left(w_{c} t+\int_{0}^{t} h(s) x(s) d s\right) & \sin \left(w_{c} t+\int_{0}^{t} h(s) x(s) d s\right) \\
-\sin \left(w_{c} t+\int_{0}^{t} h(s) x(s) d s\right) & \cos \left(w_{c} t+\int_{0}^{t} h(s) x(s) d s\right)
\end{array}\right] \\
& w(t)=\left[\begin{array}{lll}
\cos \int_{0}^{t} q^{1 / 2}(s) d w(s) & \sin \int_{0}^{t} q^{1 / 2}(s) d w(s) \\
-\sin \int_{0}^{t} q^{1 / 2}(s) d w(s) & \cos \int_{0}^{t} q^{1 / 2}(s) d w(s)
\end{array}\right]
\end{aligned}
$$

we find that

$$
d z=\left[\begin{array}{ll}
-q d t / 2 & \left(w_{c}+h x\right) d t+q^{1 / 2} d w \\
-\left(w_{c}+h x\right) d t-q^{1 / 2} d w & -q d t / 2
\end{array}\right]
$$


which is a bilinear observation equation. Similarly, $x$ and $V$ satisfy bilinear stochastic differential equations. Also, we have

$$
z(t)=x(t) v(t)
$$

-- i.e. $z$ can be thought of as a measurement of $x$ in multiplicative noise. Note that the estimation of $x$, instead of $x$, corresponds to tracking, rather than demodulating, the signal phase.

Finally, we make several comments about the matrix Lie group on which $x, v$ and $z$ evolve. This group denoted by so(2), is the group of $2 \times 2$ orthogonal matrices of determinant $+1--$ i.e.,

$$
\text { so(2) }=\left\{x \mid X^{\prime} X=I, \operatorname{det} x=+1\right\}
$$

As developed in [16] - [19], each $X \varepsilon s o(2)$ can be written in the form

$$
x=\left[\begin{array}{cc}
\cos \theta & \sin \theta \\
-\sin \theta & \cos \theta
\end{array}\right] \quad, \theta \varepsilon[-\pi, \pi]
$$

and $s o(2)$ is isomorphic (as a Lie group, [42], [43]) to the circle $s^{1}$. Also So(2) is an abelian Lie group -- i.e.

$$
X Y=Y X \quad X, Y \in S O(2)
$$

and the multiplication (3.32) corresponds to the mod $2 \pi$ addition of the corresponding angles in the representation (3.31). We note that a consequence of the commutativity of so(2) is the fact that $z$ can be written in both the bilinear form (3.28) and the multiplicative form (3.29). Example 4: As discussed in [17], the real line $\mathrm{R}^{1}$ and the circle $\mathrm{s}^{1}$ are essentially the only abelian Lie groups, since any abelian Lie group 
$G$ is isomorphic to a direct product of a number of copies of each. For instance, the group D of nonzero complex numbers is isomorphic to $\mathrm{R}^{1} \times \mathrm{S}^{1}$ under the map

$$
\begin{aligned}
& \qquad(r, \theta) \rightarrow e^{r+i \theta} \quad r \varepsilon R^{1}, \theta \varepsilon[-\pi, \pi) \\
& \text { Consider the } R^{2} \text { process } x^{\prime}=\left(x_{1}, x_{2}\right) \\
& d x(t)=A(t) x(t) d t+B(t) d w(t)
\end{aligned}
$$

where $w$ is a standard two-dimensional Brownian motion, and also consider the D-valued signal process

$$
z(t)=e^{y_{1}(t)+i y_{2}(t)}
$$

where

$$
d y(t)=x(t) d t+d v(t)
$$

Here $v$ is a 2-dimensional Brownian motion process independent of $x$. We note that $z$ satisfies a tomplex) bilinear stochastic differential equation and also that we have the multiplicative form

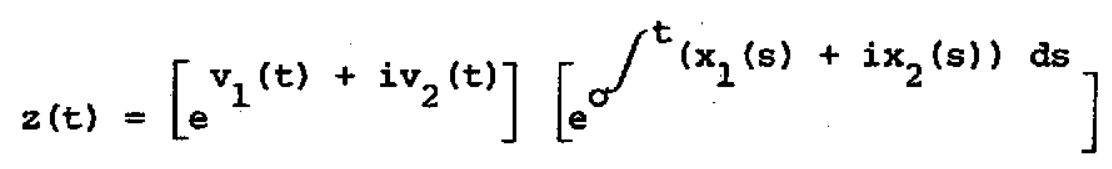

Thus $z$ is both amplitude and angle modulated, and the noise is a multiplicative-lognormal process [19], [44], including both phase and amplitude noise.

We note that the multiplicative lognormal noise process in (3.37) is an important model in some optical communication problems [44]. In many cases, changes in the transmission medium -- e.g., turbulence in the 
atmosphere - cause variations in the refractive index of the air. This disturbance can be modeled [44] as a multiplicative lognormal noise process as in (3.37). Also, if $v_{1}$ and $x_{1}$ are zero, (3.37) is identical to (3.29) if we observe that the set of unit modulus complex numbers, as well as $s o(2)$, is isomorphic to $s^{1}$. 


\section{Estimation of Rotational Processes in Three Dimensions}

As we have seen, many communication problems can be placed in the framework of estimation of processes evolving on the circle $s^{1}-$ i.e., processes of rotation in one dimension. In this section we formulate several problems of practical importance involving rotation in three dimensions. As we shall see in this and in later sections, these problems are considerably more difficult than the one dimensional problems, since rotations in three-space do not commute [13], [22], [27], [45], [46]. The problem of estimating and controlling the angular velocity and orientation of a rigid body has been studied by many authors [9], $[22],[27],[45]-[48]$ and is of great importance in many aerospace and inertial navigation applications. Such problems are by no means trivial, and most of the techniques that have been developed are suboptimal in nature. One feature of the rigid body orientation - angular velocity problem that has received some attention in the past is that the space of possible orientations is a Lie group $[22],[27],[48]$, and the combined orientation - angular velocity space is the "tangent bundle" of the orientation space and thus is a homogeneous space [49]. The framework of differential geometry and Lie theory has proven useful in studying rigid body rotation problems. In fact, there are Lie-theoretic interpretations of four of the most widely used representations of the attitude of a rigid body -- direction cosines, Euler angles, unit quaternions, and Cayley-Klein parameters.

We will consider here only the direction cosine description and refer the reader to [22] and [48] for discussions of the other representations. 
The orientation of a rigid body can be specified in terms of the direction cosines [46] between two sets of orthogonal axes -one rotating with the body and one an inertial reference frame. The direction cosines are usually given as a 3 x 3 orthogonal matrix $x$ of positive determinant -- i.e.

$$
X^{\prime} X=I \quad \operatorname{det} X=+1
$$

The set of all direction cosine matrices forms the matrix tie group So (3) [1], [16], [22]. If the 3-vector $\xi(t)$ is the (properly coordinatized, [46]) angular velocity vector of the body with respect to inertial space, the time evolution of the orientation of the body can be described by the bilinear equation

$$
x(t)=\left(\sum_{i=1}^{3} \xi_{i}(t) R_{i}\right) x(t)
$$

where $x(t) \varepsilon$ so(3) and the $R_{i}$, given by

$R_{1}=\left[\begin{array}{ccc}0 & 0 & 0 \\ 0 & 0 & -1 \\ 0 & 1 & 0\end{array}\right] \quad R_{2}=\left[\begin{array}{ccc}0 & 0 & 1 \\ 0 & 0 & 0 \\ -1 & 0 & 0\end{array}\right] \quad R_{3}=\left[\begin{array}{ccc}0 & -1 & 0 \\ 1 & 0 & 0 \\ 0 & 0 & 0\end{array}\right]$

form a basis for so(3), the matrix Lie algebra associated with so(3).

It is well known and easy to check that so(3) is not an abelian Lie group and, equivalently, that so(3) is not an abelian Lie algebra (i.e., commutator products are not identically zero). In fact so(3) is 
a simple Lie group [16], [22], [50], and it is this fact that makes the study of dynamics on $S o(3)$ so difficult. Recall that in the so(2) case we were able to represent solutions to bilinear equations by terms of. the form

$$
x(t)=\exp \left(R \int_{0}^{t} \xi(s) d s\right)
$$

where

$$
R=\left[\begin{array}{cc}
0 & 1 \\
-1 & 0
\end{array}\right]
$$

is a basis for the one-dimensional tie algebra of so(2). Wei and Norman [51], [52] have shown that one can obtain similar local expressions for the solution to equations of the form (2.1) but that such solutions are global only in certain cases -- i.e., if the underlying Lie algebra is solvable (see [10], [16], [22], and section VI of this paper for a discussion of the significance of this statement). As simple Lie algebras are not solvable, we obtain no such global representation here, and as we shall see, we must resort to suboptimal methods in the design of attitude estimation systems. We also note that the local Wei-Norman representation of the solution of (4.2) corresponds to the Euler angle description, which is well known to exist only locally (see [22] and [46], where this fact is related to the phenomenon of "gimbal-1ock"). Suppose now that the angular velocity vector in $(4.2)$ is stochastic. Specifically, we suppose $\xi$ satisfies

$$
d \xi(t)=f(t) d t+A(t) \xi(t) d t+Q^{1 / 2}(t) d w(t)
$$


where $f$ and $Q \geq 0$ are known, $\xi(0)$ is normally distributed, and $w$ is a standard 3-dimensional Brownian motion process independent of $\xi(0)$. Here f can be thought of as a vector of (known) torques acting on the body, and the Brownian motion term represents random disturbances (e.g., those caused by noisy responses of control devices, such as reaction jets, that are used to implement the desired torque $f$, or the effect of a gravity gradient).

Note also that the angular velocity equation (4.6) that we have postulated is simpler than the usual nonlinear Euler equations [46]. Equation (4.6), with suitable choice of $f, A$, and $Q$, can be viewed as a reasonable approximation if: (1) the rigid body is nearly spherically symetric (so that the principle moments of inertia are almost equal -in this case the nonlinear terms in the Euler equations are small and may be lumped into the random disturbance term); or (2) we linearize Euler's equations about a nominal (which might be included in the $f(t)$ term); or (3) we make $Q(t)$ large enough so that the nonlinear effects can be viewed as pracess noise.

We now can describe two different measurement processes -one motivated by a strapdown navigation system, and the other by an inertial system in which a platform is to be kept inertially fixed. In a strapdown system, [46] one receives noisy information about either angular velocity or incremental angle changes. Assuming that the size of the increment is small, either type of information can be modeled by the I to equation

$$
d z(t)=c(t) \xi(t) d t+s^{1 / 2}(t) d v(t)
$$


where $\mathrm{S}>0$ and $\mathrm{v}$ is a standard Brownian motion process, independent of . This equation is the precise analog of the usual (formal) observation equation (for rate gyroscopes)

$$
\dot{z}(t)=c(t) \xi(t)+\dot{v}(t)
$$

Here $\dot{v}$ is white gyro noise. Another possible observation model is provided by integrating gyroscopes, in which case we observe

$$
m(t)=c(t) \int_{0}^{t} \xi(s) d s+N(t)
$$

Here $\mathrm{N}$ represents a gyro drift. Usually when using a model like (4.9), one assumes that the drift is a correlated process. This adds no real difficulty to the problem, since there are simple techniques for handing measurements with additive colored noise [53]. In fact, the consideration of the process (4.9) rather than (4.8) adds no difficulty to the analysis, and thus we will concentrate on $(4.8)$.

In the usual strapdown system the information (4.7) is processed by a "direction cosine computer," which produces $z(t)$, the solution of

$$
d z(t)=\left[\sum_{i=1}^{3} R_{i} d z_{i}(t)+\frac{1}{2} \sum_{i=1}^{3} \sum_{j=1}^{3} s_{i j}(t) R_{i} R_{j} d t\right] z(t)
$$

our problem is to use the information supplied by $z$ or $z$ to compute "good" estimates of the angular velocity $\xi$ and the orientation $x$. Thus, if we take (4.7) as our basic measurement equation, we have linear observations, while (4.10) yields a bilinear measurement process.

A second type of observation process is suggested by an inertial system equipped with a platform that is to "instrument" (i.e. remain fixed 
with respect to) the inertial reference frame. Suppose we use the notation b-frame for the body-fixed frame, p-frame for the platform frame, and $i$-frame for the inertial reference frame. Letting $c_{\alpha}^{\beta}(t)$ denote the direction cosine matrix of the $\beta$-frame with respect to the $\alpha$-frame, we have

$$
x(t)=c_{i}^{b}(t)
$$

Also, by noting the relative orientation of the platform and the body (perhaps by reading of gimbal angles [46], we can measure

Let

$$
M(t)=C_{p}^{b}(t)
$$

$$
v(t)=c_{i}^{p}(t)
$$

Then $v(t)$ represents platform misalignment -- a drift of the platform with respect to inertial space due both to drifts in the gyroscopes used to sense rotation of the rigid body and also to inaccuracies in the mechanism that rotates the platform relative to the body in order to keep it inertially fixed. If we model gyro drifts and the other inaccuracies as Brownian motion processes, a reasonable model for $\mathrm{V}$ is as an so(3) Brownian motion, [13], [16], [22], [54]:

$$
d v(t)=\left[\sum_{i=1}^{3} R_{i} d v_{i}(t)+\frac{1}{2} \sum_{i=1}^{3} \sum_{i=1}^{3} s_{i j}(t) R_{i} R_{j} d t\right] v(t)
$$


where $v$ is the Brownian motion process with $E\left[d v(t) d v^{\prime}(t)\right]=s(t) d t$. Using elementrary properties of direction cosine matrices [46], we have

$$
M(t)=C_{p}^{b}(t)=c_{i}^{b}(t) C_{p}^{i}(t)=c_{i}^{b}(t)\left[c_{i}^{b}(t)\right]^{\prime}=x(t) V^{\prime}(t) .
$$

(multiplicative observation noise) and, using (4.2) and (4.14) (plus the fact that $R_{i}^{\prime}=-R_{i}$ ), we have the following stochastic differential equation for M:

$d M(t)=\left[\sum_{i=1}^{3} R_{i} \xi_{i}(t)\right] M(t) d t+M(t)\left[-\sum_{i=1}^{3} R_{i} d v_{i}(t)+\frac{1}{2} \sum_{i, j=1}^{3} s_{i j}(t) R_{i} R_{j} d t\right]$

Again, we wish to consider the problem of estimating $\xi$ and $\mathrm{X}$ given the observation process $M$.

In many inertial systems the type of information that is available is in the form of "pulses" [46] from the gyros. If this is the case, it is logical to take the incremental equation $(4.10)$ as the basic sensor equation. Also, for the second observation process, if we take the incremental gimbal angles as the quantity we observe, equation (4.16) becomes the basic measurement equation.

Now consider (4.2) with the $3 \times 3$ matrix $x$ replaced by the 3-vector $x$. Assuming that $x^{\prime}(0) x(0)=1$, we have that $x^{\prime}(t) x(t)=1, \forall t--i . e$. , $x$ evolves on $s^{2}$, the unit sphere in 3-space. The study of random processes with constant "energy" is of importance in a number of fields -- including DC to DC conversion [55], statistical mechanics [56], and satellite orbital analysis [16], [57], [58]. We briefly describe a simplified version of 
a satellite tracking problem of great practical importance.

Consider a satellite in circular orbit about some celestial body, such as the moon. Because of a variety of effects including anomalies in the gravitational field of the body, effects of the gravitational fields of nearby bodies, and the effects of "solar wind", the orbit of the satellite is perturbed. For simplicity, we assume that the perturbations of the orbit are tangential only -- i.e. the radial effects are unimportant or have been corrected for. In this case, a stochastic version of (4.2) arises. Suppose the angular velocity $\xi$ of the vehicle with respect to body can be written the form

$$
\xi(t)=f(t)+\dot{w}(t)
$$

where $f$ is the nominal orbit ("carrier") angulary velocity and $\dot{w}$ is a white perturbation with

$$
E[\dot{w}(t) \dot{w}(s)]=Q(t) \delta(t-s)
$$

The stochastic analog of (4.2) then is

$$
\begin{aligned}
d x(t)=\left\{\left[\sum_{i=1}^{3} f_{i}(t) A_{i}\right.\right. & \left.+\sum_{i, j=1}^{3} Q_{i j}(t) A_{i} A_{j}\right] d t \\
& \left.+\sum_{i=1}^{3} A_{i} d w_{i}(t)\right\} x(t)
\end{aligned}
$$

If we are then given noisy observations of the satellite position

$$
d z(t)=H(t) x(t) d t+R^{1 / 2}(t) d v(t)
$$

where $v$ is Brownian observation noise independent of $x$ and $R(t)>0$, our problem is to estimate $x(t)$. 
We note that the assumption that the angular velocity perturbations are white is a simplification. For instance, the anomalies in the moon's gravitational field are spatially correlated and constitute a random field [29], [57], [58], which can be estimated [58] from the observed data (4.20). However, the computations involved in such an estimation are of such magnitude that they must be computed off-line. The simpler model (4.19) may lead to simple but accurate on-line tracking schemes (see Section VII). Also, by including perturbations with time correlation consistent with the period of the orbit, we may be able to make estimation systems based on (4.19), (4.20) "smarter" with only minor complications in tracking system design. We note that $(4.19),(4.20)$ is a direct $s^{2}$ analog of the $s^{1}$ incoherent oscillator tracking problem described in Example 1 (incoherent orbiters?).

In fact, we can again use the constrained least-squares criterion: minimize

$$
E\left[(\dot{x}(t)-\widetilde{x}(t))^{\prime}(x(t)-\widetilde{x}(t)) \mid z(s), 0 \leq s \leq t\right]
$$

subject to

$$
\|\widetilde{x}(t)\|^{2}=\tilde{x}^{\prime}(t) \widetilde{x}(t)=1
$$

It can be shown that, as in the $s^{1}$ problem,

$$
x(t)=\frac{\hat{x}(t \mid t)}{\|\hat{x}(t \mid t)\|}
$$




\section{v. Estimation of Air Pollution}

The problem of estimating the concentration of pollutants in the air is a vital first step toward the goal of maintaining the air pollution levels within safe limits. A recently developed stochastic model for air pollution [63], [64] involves partial differential equations which, when discretized, become discrete-time bilinear equations (see [65], [66] for discussions of discrete-time bilinear systems). The advection-diffusion model of [63], [64], which is a generalization of the widely-used steady-state Gaussian plume model, accounts for the continuous fluctuation of meteorological factors by means of stochastic modeling.

The transportation in air of a single-species pollutant is approximated by the advection-diffusion equation

$$
\begin{aligned}
\frac{\partial C}{\partial t}(x, y, z, t)= & -\bar{v} \cdot \nabla c(x, y, z, t)+\nabla\left(k_{c} \nabla c(x, y, z, t)\right) \\
& +\bar{Q}_{c}(x, y, z, t)-v \cdot \nabla \bar{c}(x, y, z, t)+q_{c}(x, y, z, t)
\end{aligned}
$$

where $C$ is the pollution concentration; $\bar{C}$ is the mean of $c ; \bar{v}$ and $v$ are the mean and the zero-mean stochastic component of the wind velocity; $\bar{Q}_{c}$ and $q_{c}$ are the mean and the zero-mean stochastic component of the pollution source rate; and $\mathrm{k}_{\mathrm{c}}$ is the eddy diffusivity. For the boundary conditions associated with (5.1), see [63], [64].

For practical implementation, the advection-diffusion equation (5.1) is discretized both in space and time, resulting in the finite dimensional system of equations 
$A x(t+1)=\left[2 I-A+\hat{E}\left(V_{D}\right)\right] x(t)+\bar{D}+L \bar{Q}_{C}+\Gamma(x(t)) w(t)$

$$
\begin{aligned}
& \Gamma(x(t))=[G(x(t)) \vdots L] \\
& w(t)=\left[\begin{array}{l}
v(t) \\
q_{C}(t)
\end{array}\right] \\
& z(t)=H x(t)+\xi(t)
\end{aligned}
$$

where $x(t)$ is the vector of pollution concentrations in the cells of the spatial discretization, and $A, L, \hat{E}\left(V_{D}\right), \bar{D}, G, H$, and $I(=$ identity) are known constant matrices. The noise processes $v(t), q_{C}(t)$, and $\xi(t)$ are assumed to be independent, Gaussian, and white. Since $\Gamma(x(t))$ is linear in $x(t)$, the system (5.2) is obviously of the discrete-time bilinear form, and it is driven by fluctuations in the wind velocity and source rate.

We will not consider specific estimation schemes for the system (5.2) - (5.3); however, we remark that the cumulant method of section VIII may provide a useful suboptimal approach to the problem. In [63], [64] it is assumed that $\Gamma(x(t))$ is slowly varying, and a suboptimal filter, which employs a non-Riccati estimation algorithm using incremental covariance [67], [68], is designed for the resulting "linear" system. Finally, we refer the reader to [80] for a discussion of deterministic bilinear systems described by partial differential equations. Also, if we discretize in space only, we obtain continuous-time bilinear equations, and the techniques of Sections VI and VII may be applicable. 


\section{Finite Dimensional Optimal Nonlinear Estimators}

In this section we will consider the estimation problem for a class of systems evolving on nilpotent or solvable Lie groups [10], [16], [22], [69]. The equations we will consider are motivated by the strapdown navigation system of system IV --

$$
\begin{aligned}
d \xi(t) & =F(t) \xi(t) d t+o^{1 / 2}(t) d w(t) \\
x(t) & =\left(A_{0}+{ }_{i=1}^{n} \xi_{i}(t) A_{i}\right) x(t) ; x(0)=I \\
d z(t) & =H(t) \xi(t) d t+R^{1 / 2}(t) d v(t)
\end{aligned}
$$

where $\xi(t)$ is an n-vector, $x(t)$ is a $k x$ matrix, $z(t)$ is a p-vector, $w$ and $v$ are independent standard Brownian motion processes, $Q \geq 0$, $R>0$, and $\xi(0)$ has a Gaussian density independent of $w$ and $v$. The criterion for the optimal estimate $\tilde{x}(t \mid t)$ will be the minimization of

$$
E[\operatorname{tr}\{(x(t)-\tilde{x}(t)),(x(t)-\tilde{x}(t))\} \mid z(s), 0 \leq s \leq t\},
$$

where "tr" denotes trace. It is well known [28] that this minimum-variance estimate is given by the conditional mean

$$
\hat{x}(t \mid t)=E^{t}[x(t)] \triangleq E[X(t) \mid z(s), 0 \leq s \leq t\},
$$

In general, the computation of $\hat{x}(t \mid t)$ requires an infinite-dimensional system of equations, and approximations must be made for practical implementation (see the related comments in [28] and sections VII and VIII). Thus it is of interest to study systems for which the optimal minimum variance estimator is dimensional (and thus implementable on-line with a digital computer).

$$
-28-
$$


Throughout this section, we assume that $\left\{A_{i}\right\}_{L A^{\prime}}$ the smallest Lie algebra which contains $\left\{A_{i}, i=0,1, \ldots, n\right\}$, is solvable; this is equivalent to the existance of a complex nonsingular $k \times k$ matrix $P$ such that, for all elements $A$ in $\left\{A_{i}\right\}_{L A}$, the matrix $P A^{-1}$ is in the upper triangular form where $a_{i j}=0$ for $i>j$ and the other elements are arbitrary (see [69] for further details on solvable Lie groups and Lie algebras). Thus we will assume that the matrices $\left\{A_{i}\right\}$ in (6.2) are in upper triangular form; this implies that $x$ evolves on the solvable Lie group $G_{s}(k)$ of nonsingular upper triangular $\mathrm{k} \times \mathrm{k}$ matrices.

First we consider the Lie subgroup $G_{N}(k)$ of upper triangular $k \times k$ matrices with $a_{i j}=1, i=1, \ldots, k$; this is a nilpotent Lie group. The corresponding nilpotent Lie algebra $I_{N}(k)$ consists of the strictly upper triangular $k \times k$ matrices. In this case, because the Peano-Baker series is finite, $x(t)$ can be expressed in closed form in terms of a finite number of integrals in which the integrands are products of the components of $\xi$. We can show that, for the system (6.1)-(6.3) evolving on $G_{N}(k)$, the optimal estimate $\hat{x}(t \mid t)$ can be computed by a finite dimensional nonlinear filter. The starting point of the derivation is a closed-form expression for $\mathrm{x}(t)$.

For simplicity, the filter will only be derived for $n=k=3$, but the result can also be proved by induction for higher-order systems. Let $A_{0}=0$ and

$A_{1}=\left[\begin{array}{lll}0 & 1 & 0 \\ 0 & 0 & 0 \\ 0 & 0 & 0\end{array}\right] \quad A_{2}=\left[\begin{array}{lll}0 & 0 & 1 \\ 0 & 0 & 0 \\ 0 & 0 & 0\end{array}\right] \quad A_{3}=\left[\begin{array}{lll}0 & 0 & 0 \\ 0 & 0 & 1 \\ 0 & 0 & 0\end{array}\right]$

Then $\left\{A_{1}, A_{2}, A_{3}\right\}$ is a basis for $L_{N}(3)$. The solution of (6.2) can be expressed in closed form as 
$x(t)=\left[\begin{array}{ccc}1 & \int_{0}^{t} \xi_{1}(\sigma) d \sigma & \int_{0}^{t} \xi_{2}(\sigma) d \sigma+\int_{0}^{t} \int_{1}^{\sigma_{1}} \xi_{1}\left(\sigma_{1}\right) \xi_{3}\left(\sigma_{2}\right) d \sigma_{2} d \sigma \\ 0 & 1 & \int_{0}^{t} \xi_{3}(\sigma) d \sigma \\ 0 & 0 & 1\end{array}\right]$

Before stating the major theorem concerning the computation of $\hat{x}(t \mid t)$, we need two preliminary results.

Lemma 6.1: Consider the linear system $(6.1),(6.3)$, and define (for $\sigma \leq t)$

$$
E^{t}[\xi(\sigma)]=\hat{\xi}(\sigma \mid t)=E[\xi(\sigma) \mid z(s), 0 \leq s \leq t]
$$

Then the conditional cross-covariance matrix

$\left.P\left(\sigma_{1}, \sigma_{2}, t\right)=E\left[\xi\left(\sigma_{1}\right)-\hat{\xi}\left(\sigma_{1} \mid t\right)\right)\left(\xi\left(\sigma_{2}\right)-\hat{\xi}\left(\sigma_{2} \mid t\right)\right)^{-} \mid z(s), 0 \leq s \leq t\right]$

is nonrandom -- i.e., it is independent of $\{z(s), 0 \leq s \leq t\}$.

The proof of Lemma 6.1 is based upon the fact that the error covariance matrices for the linear Gaussian estimation and smoothing problems are nonrandom [71]. This lemma allows the off-line computation of $P\left(\sigma_{1}, \sigma_{2}, t\right)$ via Kwakernaak's equations [72] (for $\sigma_{1} \leq \sigma_{2}$ )

$$
\begin{aligned}
P\left(\sigma_{1}, \sigma_{2}, t\right) & =P\left(\sigma_{1}\right) \Psi^{\prime}\left(\sigma_{2}, \sigma_{1}\right) \\
& -P\left(\sigma_{1}\right)\left[\int_{\sigma_{2}}^{t} \Psi^{\prime}\left(\tau, \sigma_{1}\right) H^{\prime}(\tau) R^{-1}(\tau) H(\tau) \Psi\left(\tau, \sigma_{2}\right) d \tau\right] P\left(\sigma_{2}\right)
\end{aligned}
$$


$\frac{d}{d t} \Psi(t, \tau)=\left[F(t)-P(t) H^{\prime}(t) R^{-I}(t) H(t)\right] \Psi(t, \tau) ; \Psi(\tau, \tau)=I$

where the Kalman filter error covariance matrix $P(t)=P(t, t, t)$ is computed by the Riccati equation

$$
\dot{P}(t)=F(t) P(t)+P(t) F^{\prime}(t)+Q(t)-P(t) H^{\prime}(t) R^{-1}(t) H(t) P(t)
$$

Lemma 6.2: The conditional cross-covariance satisfies

$$
P(\sigma, t, t)=K(t, \sigma) P(t)
$$

where

$\frac{d}{d t} K^{\prime}(t, \sigma)=-\left[F^{\prime}(t)+P^{-1}(t) Q(t)\right] K^{\prime}(t, \sigma) ; K^{\prime}(\sigma, \sigma)=I$

This lemma follows easily from some identities in [73]. Equation (6.12) implies that $K^{\prime}(t, \sigma)$ is a transition matrix satisfying the semigroup property

$$
K^{\prime}(t, \sigma)=K^{\prime}(t,-s) K^{\prime}(s, \sigma)
$$

These properties of $\mathrm{P}(\sigma, t, t)$ are crucial in the derivation of the next theorem, which states the major result concerning the computation of $x(t \mid t)$.

Theorem 6.1: Consider the system of equations (6.1) - (6.4). The conditional expectation $\hat{x}(t \mid t)$ is generated by the following finitedimensional nonlinear filter. First, augment the state of the linear equation (6.1) by writing 


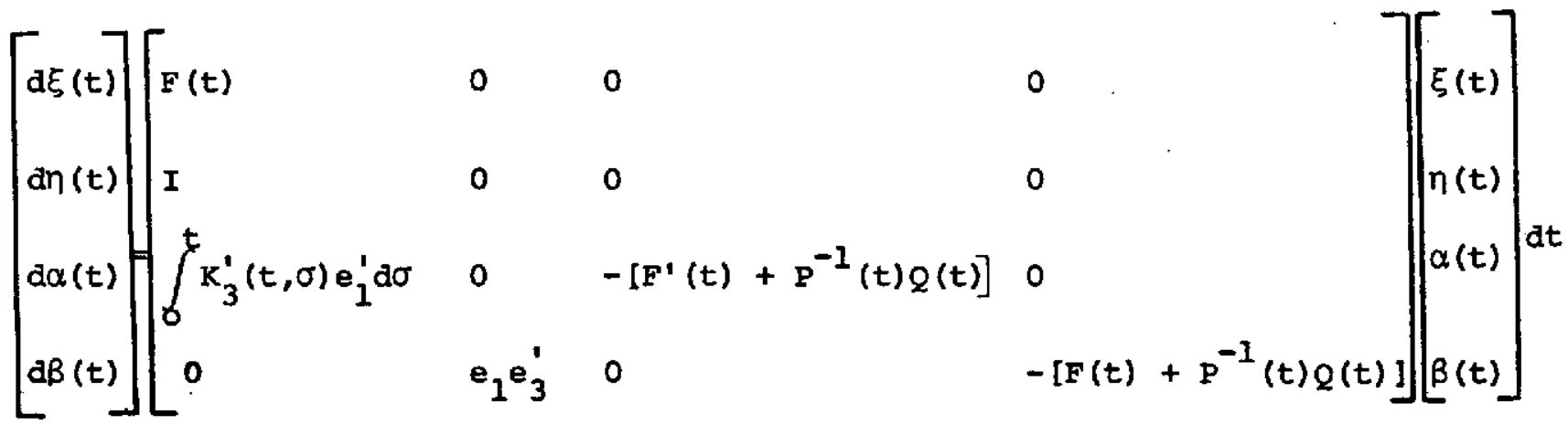

$$
\begin{aligned}
& +\left[\begin{array}{c}
Q^{1 / 2}(t) \\
0 \\
0 \\
0
\end{array}\right] d w(t) \\
& n(0)=\alpha(0)=\beta(0)=0 \\
& d z(t)=\left[\begin{array}{llll}
H(t) & 0 & 0 & 0
\end{array}\right]\left[\begin{array}{l}
\xi(t) \\
\eta(t) \\
\alpha(t) \\
\beta(t)
\end{array}\right] d t+R^{1 / 2}(t) d v(t)
\end{aligned}
$$

where $k(t, \sigma)$ is given by $(6.12)$, and $P(t)$ is given by $(6.10)$. Here

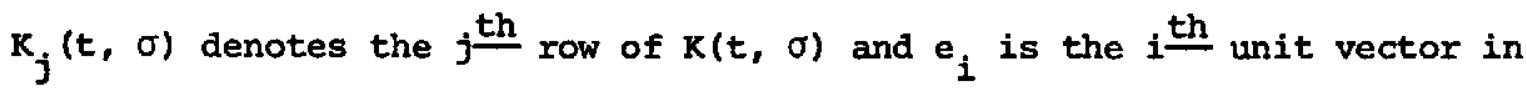
$R^{3}$

Then the Kalman filter [28] for $(6.14)-(6.16)$ yields the conditional expectations $\hat{\xi}(t \mid t), \hat{\eta}(t \mid t), \hat{\alpha}(t \mid t)$, and $\hat{\beta}(t \mid t)$. The conditional expectation $\hat{x}(t \mid t)$ is computed by

$$
\hat{x}(t \mid t)=\left[\begin{array}{ccc}
1 & \hat{n}_{1}(t \mid t) & \hat{n}_{2}(t \mid t)+\hat{\gamma}(t \mid t) \\
0 & 1 & \hat{n}_{3}(t \mid t) \\
0 & 0 & 1
\end{array}\right]
$$


where

$$
\begin{aligned}
d \hat{\gamma}(t \mid t) & =\left[\hat{\xi}_{1}(t \mid t) \hat{n}_{3}(t \mid t)+\int_{0}^{t} P_{13}(t, \sigma, t) d \sigma\right] d t \\
& +\left[\hat{\alpha}^{\prime}(t \mid t)+\hat{B}^{\prime}(t \mid t)\right] P(t) H^{\prime}(t) R^{-1}(t)[d z(t)-H(t) \hat{\xi}(t \mid t) d t] \\
& \gamma(0 \mid 0)=0
\end{aligned}
$$

Proof: All the terms in (6.17) result from linear filtering theory [28], except for $\hat{\gamma}(t \mid t)$. In the derivation of (6.18) we will frequently use a version of Fubinis theorem [74] which allows us to interchange integrals with conditional expectations. Notice that

$$
\hat{\gamma}(t \mid t)=E^{t}\left[\int_{0}^{t} \int_{0}^{\sigma_{1}} \xi_{1}\left(\sigma_{1}\right) \xi_{3}\left(\sigma_{2}\right) d \sigma_{2} d \sigma_{1}\right]
$$

Thus if we define $\gamma$ by

$$
\begin{aligned}
d \gamma(t) & =\left[\xi_{1}(t) \int_{0}^{t} \xi_{3}(\sigma) d \sigma\right] d t \\
& =\xi_{1}(t) \eta_{3}(t) d t \\
\gamma(0) & =0
\end{aligned}
$$

then Kushner's equation [28] yields the conditional expectation

$$
\begin{aligned}
\hat{d \gamma}(t \mid t) & =E^{t}\left[\xi_{1}(t) \eta_{3}(t)\right] d t \\
& +\left\{E^{t}\left[\gamma(t) \xi^{\prime}(t)\right]-\hat{\gamma}(t \mid t) \hat{\xi}(t \mid t)\right\} H^{\prime}(t) R^{-1}(t)[d z(t)-H(t) \hat{\xi}(t \mid t) d t]
\end{aligned}
$$

From the definition of $\mathrm{P}\left(\sigma_{1}, \sigma_{2}, t\right)$ it follows that 
$E^{t}\left[\xi_{1}(t) \eta_{3}(t)\right]=\hat{\xi}_{1}(t \mid t) \hat{n}_{3}(t \mid t)+\int_{0}^{t} P_{13}(t, \sigma, t) d \sigma$

Considering the second term in (6.21),

$$
\begin{aligned}
& E^{t}\left[\gamma(t) \xi_{i}(t)\right]-\hat{\gamma}(t \mid t) \xi_{i}(t \mid t) \\
& =\int_{0}^{t} \int_{0}^{1}\left\{E^{t}\left[\xi_{1}\left(\sigma_{1}\right) \xi_{3}\left(\sigma_{2}\right) \xi_{i}(t)\right]-\hat{\xi}_{i}(t \mid t) E^{t}\left[\xi_{1}\left(\sigma_{1}\right) \xi_{3}\left(\sigma_{2}\right)\right]\right\} d \sigma_{2} d \sigma_{1} \\
& \quad=\int_{0}^{t} \int_{0}^{g}\left\{P_{1 i}\left(\sigma_{1}, t, t\right) E^{t}\left[\xi_{3}\left(\sigma_{2}\right)\right]+P_{3 i}\left(\sigma_{2}, t, t\right) E^{t}\left[\xi_{1}\left(\sigma_{1}\right)\right]\right\} d \sigma_{2} d \sigma_{1}
\end{aligned}
$$

where the last term in $(6.23)$ is a result of the definition of $P\left(\sigma_{1}, \sigma_{2}, t\right)$ and the expansion of the third order moment of a Gaussian distribution [75].

Thus by Lemmas 6.1 and 6.2

$E^{t}\left[\gamma(t) \xi^{\prime}(t)\right]-\hat{\gamma}(t \mid t) \hat{\xi}^{\prime}(t \mid t)$

$=\int_{0}^{t} \int_{0}^{\sigma l}\left\{k_{1}\left(t, \sigma_{1}\right) E^{t}\left\{\xi_{3}\left(\sigma_{2}\right)\right]+K_{3}\left(t, \sigma_{2}\right) E^{t}\left[\xi_{1}\left(\sigma_{1}\right)\right\}\right\} P(t) d \sigma_{2} d \sigma_{1}$

$=E^{t}\left[\int_{0}^{t} x_{1}\left(t, \sigma_{1}\right) \eta_{3}\left(\sigma_{1}\right) d \sigma_{1}+\int_{0}^{t} \xi_{1}\left(\sigma_{1}\right) \int_{0}^{\sigma_{1}} x_{3}\left(t, \sigma_{2}\right) d \sigma_{2} d \sigma_{1}\right] P(t)$

$=\left(E^{t}\left[\beta^{\prime}(t)\right]+E^{t}\left[\sigma^{\prime}(t)\right]\right) P(t)$

The fact that equation (6.14) for $\alpha$ and $\beta$ provides a realization of the argument in (6.24) is a direct consequence of (6.12)

Further insight into the structure of the optimal nonlinear filter of Theorem 6.1 is provided by the equivalent formulation 


$$
\left.\begin{array}{l}
d \hat{x}(t \mid t)=\left\{\left(\sum_{i=1}^{3} A_{i} \hat{\xi}_{i}(t \mid t)\right) \hat{x}(t \mid t)+E_{13} \int_{13}^{t} P_{13}(t, \sigma, t) d \sigma\right\} d t \\
\left.+\left\{\int_{0}^{t}\left[\begin{array}{lrr}
0 & k_{1}(t, \sigma) & k_{2}(t, \sigma) \\
0 & 0 & k_{3}(t, \sigma) \\
0 & 0 & 0
\end{array}\right]+\hat{\alpha} \cdot(t \mid t)+\hat{\beta}^{\prime}(t \mid t)\right) E_{13}\right\} \\
. P(t) H^{\prime}(t) R^{-1}(t)[d z(t)-H(t) \hat{\xi}(t \mid t) d t]
\end{array}\right\}
$$

where $E_{i j}$ has a 1 as its $(i, j)$ th element and zeros elsewhere: Thus the filter for $\hat{x}(t \mid t)$ contains a model of the original system (6.2) driven by the innovations process $d \nu(t)=d z(t)-H(t) \hat{\xi}(t \mid t)$ and the outputs of a linear filter (see the block diagram in Figure 6.1).

In addition to providing the optimal minimum variance estimate, it can be shown that the filter of Theorem 6.1 contains fewer states than the extended Kalman filter [28] for the same system. This is due to the on-line computation of the "approximate" error covariance matrix in the extended Kalman filter.

Theorem 6.1 can be extended in various ways. First, it can be extended to systems of the form $(6.1),(6.3)$, and

$$
\dot{Y}(t)=\left[\begin{array}{ccc}
a_{1}(t) & \xi_{1}(t) & \xi_{2}(t) \\
0 & a_{2}(t) & \xi_{3}(t) \\
0 & 0 & a_{3}(t)
\end{array}\right] \quad Y(t) ; Y(0)=I
$$

with nonrandom diagonal terms $a_{i}$; such systems evolve on the solvable Lie group $G_{s}(3)$. Second, the results are valid for systems evolving on 


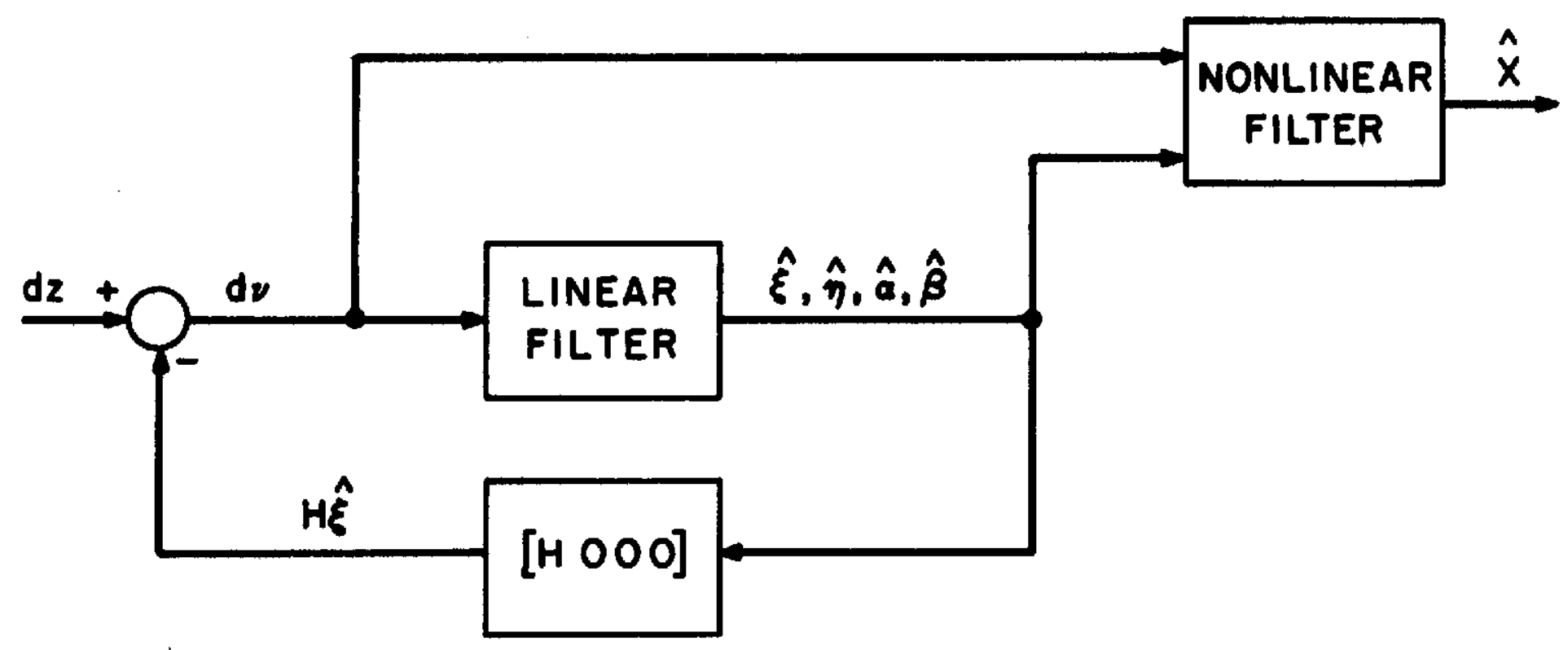

Fig. 6.1 Block Diagram for Optimal Nonlinear Filtering on $G_{N}(3)$ 
$G_{N}(k)$ and for systems with nonrandom diagonal terms evolving on $G_{S}(k)$.

Finally, the results may be extended to observations of the form of $(2.12)$

or (2.13); in these cases, it can be shown [20], [26] that the observations

are, in a certain sense, "conditionally" linear. For all of these ex-

tensions of Theorem 6.1, it can be shown that the optimal minimum

variance filter is finite dimensional. 
VII. The Use of Harmonic Analysis in Suboptimal Filter Design

A very important result of harmonic analysis states that the eigenfunctions of the Laplace-Betrami operator on a compact manifold $M$ are a complete set of functions in $I^{2}(M, \mu)$ (where $\mu=$ Haar measure) [43], [76]. In this section we will develop suboptimal estimation techniques for bilinear systems evolving on compact manifolds by employing an approximation to the conditional density which is based on these eigenfunctions.

First consider Example 1 of Section III which evolves on the Lie group $s^{1}$, described by $(3.4)-(3.5)$ or $(3.7)-(3.8)$. As discussed in [24], the optimal (constrained least-squares) filter is described as follows. The conditional probability density of $\theta$ given $\{z(s), 0 \leq s \leq t\}$ may be expanded in the Fourier series (notice that the trigonometric polynomials are eigenfunctions of the Laplacian on $s^{l}$ )

$$
p(\theta, t)=\sum_{n=-\infty}^{+\infty} c_{n}(t) e^{i n \theta}
$$

where

$$
\begin{aligned}
c_{n}(t) & =\frac{1}{2 \pi} E\left[e^{-i n \theta(t)} \mid z(s), 0 \leq s \leq t\right] \\
& =b_{n}(t)-i a_{n}(t)
\end{aligned}
$$

Then

$$
\begin{aligned}
d c_{n}(t) & =-\left[i n w_{c}+\frac{n^{2}}{2} q(t)\right] c_{n}(t) d t \\
& +\left[\frac{\left(c_{n-1}(t)-c_{n+1}(t)\right)}{2 i}+2 \pi c_{n}(t) \operatorname{Im}\left(c_{1}(t)\right)\right]\left[\frac{d z(t)+2 \pi \operatorname{Im}\left(c_{1}(t)\right) d t}{r(t)}\right]
\end{aligned}
$$




$$
\tilde{\theta}(t)=\tan ^{-1}\left(a_{1}(t) / b_{1}(t)\right)
$$

Since $c_{0}=\frac{1}{2 \pi}$ and $c_{-n}=c_{n}^{*}$ (where * denotes the complex conjugate). we need only solve (7.3) for $n \geq 1$. The structure of the optimal filter, which is illustrated in Figures 7.1 and 7.2 , deserves further comment (recall that $\left.c_{n}=b_{n}-i a_{n}\right)$. The filter consists of an infinite bank of filters, the nth of which is essentially a damped oscillator, with oscillator frequency $\mathrm{nw}_{\mathrm{c}}$ ' together with nonlinear couplings to the other filters and to the received signal. Notice, however, that the equation for $c_{n}$ is coupled only to the filters for $c_{1}, c_{n-1}$, and $c_{n+1}$. This fact will play an important part in our approximation.

In order to construct a finite-dimensional suboptimal filter, we wish to approximate the conditional density (7.1) by a density determined by a finite set of parameters. Several examples of "assumed density" approximations for this problem are discussed in [16], [25], but the most useful involves the assumption that $p(\theta, t)$ is a folded normal density (see [16], [17], [24]) with mode $n(t)$ and "variance" $\gamma(t)$ :

$$
p(\theta, t)=\frac{1}{2 \pi} \sum_{n=-\infty}^{+\infty} e^{-n^{2} \gamma(t) / 2} e^{i \dot{n}(\theta-n(t))}=F(\theta ; n(t), \gamma(t))
$$

This density is related to the normal density in the following way: if $x$ is a real random variable with density $N(\alpha ; \eta, \gamma)$, then $\theta=x \bmod 2 \pi$ has the density

$$
F(\alpha ; n, \gamma)=\sum_{n=-\infty}^{+\infty} N(\alpha+2 n \pi ; n, \gamma)
$$




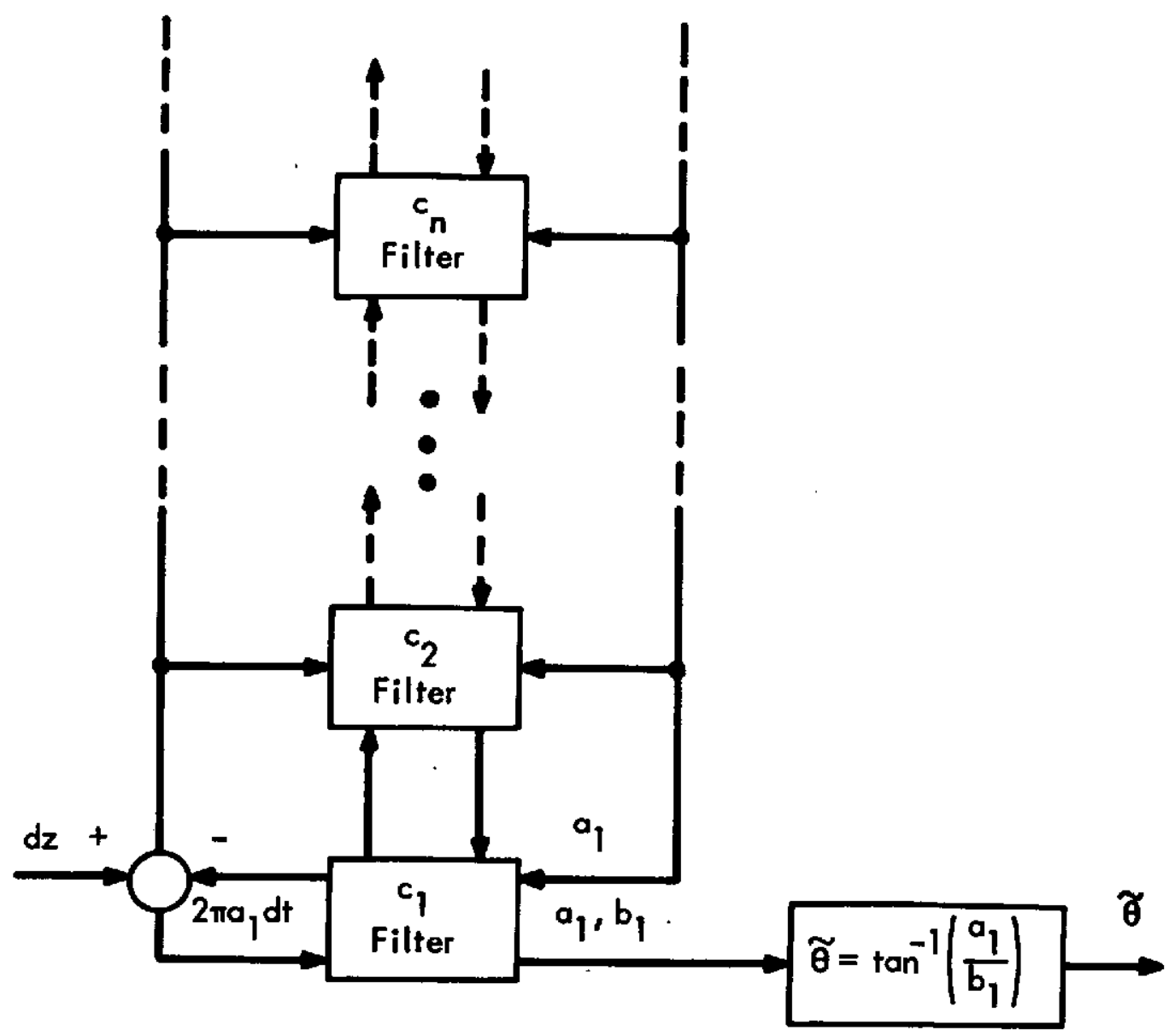

Fig. 7.1 Illustrating the Form of the Infinite Dimensional Optimal Filter of Example 1, Section III 


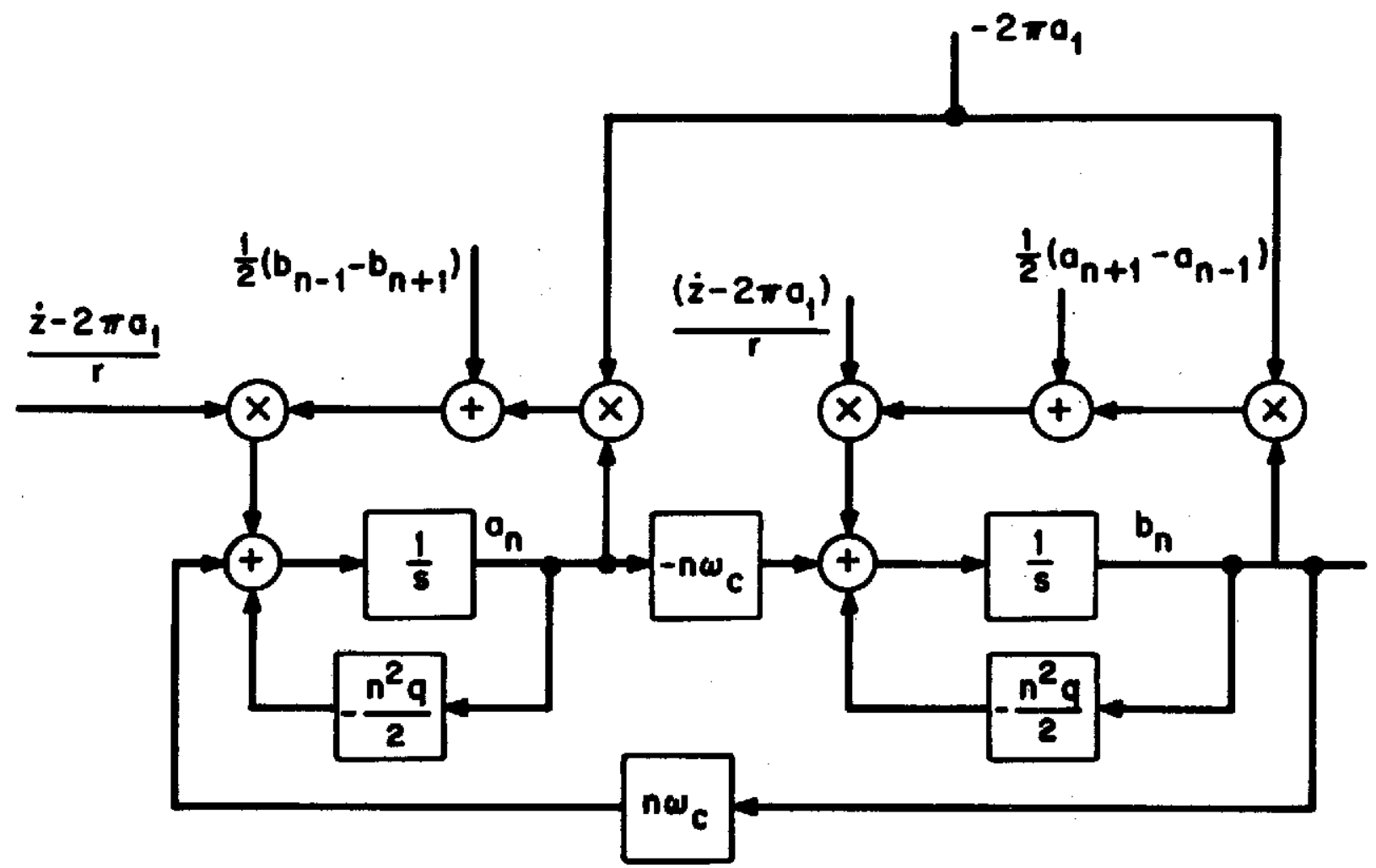

Fig. 7.2 Diagram of the $c_{n}$ Filter shown in Fig. 7.1 
--i.e., we "fold" $N(\alpha ; \eta, \gamma)$ around the circle. We note that the folded normal density is the solution of the standard diffusion equation on the circle (i.e., it is the density for $s^{1}$ Brownian motion processes) and is as important a density on $\mathrm{s}^{1}$ as the normal is on $\mathrm{R}^{1}$.

In this case, if $c_{1}$ has been computed and if $p(\theta, t)$ satisfies (7.5), then $\mathrm{C}_{\mathrm{N}+1}$ can be computed (for any $\mathrm{N}$ ) from the equation

$$
c_{N+1}=(2 \pi)^{(N+1)^{2}-1}\left|c_{1}\right|^{N(N+1)} c_{1}^{(N+1)}
$$

Thus we can truncate the bank of filters described by (7.3) by approximating $c_{\mathrm{N}+1}$ by (7.7) and substituting this approximation into the equation for $\mathrm{C}_{\mathrm{N}}$. This was done for $\mathrm{N}=1$ in $[25]$; the resulting suboptimal filter equations are

$$
\begin{gathered}
\dot{a}_{1}=\left(w_{c} b_{1}-\frac{g}{2} a_{1}\right)+\frac{\left(\dot{z}-2 \pi a_{1}\right)}{r}\left[\frac{1}{2}\left(\frac{1}{2 \pi}-8 \pi^{3}\left(b_{1}^{4}-a_{1}^{4}\right)-2 \pi a_{1}^{2}\right]\right. \\
\dot{b}_{1}=-\left(w_{c} a_{1}+\frac{g}{2} b_{1}\right)+\frac{\left(\dot{z}-2 \pi a_{1}\right)}{r}\left[8 \pi^{3} a_{1} b_{1}\left(a_{1}^{2}+b_{1}^{2}\right)-2 \pi a_{1} b_{1}\right] \\
\tilde{\theta}=\tan ^{-1}\left(a_{1} / b_{1}\right)
\end{gathered}
$$

In [25], this Fourier coefficient filter (FCF) was compared to a phaselock loop [32] and to the Gustafson-Speyer "state-dependent noise filter" (SDNF) [34]. The FCF performed consistently better than the other systems, although the SDNF performance was quite close.

Similar analyses and "assumed density" approximations can be applied to the other examples in Section III; the reader is referred to 
[25], [28], [35], and Section VIII of this paper.

Motivated by the success of the previous example evolving on $s^{l}$, we will now extend these results to the system (4.19) - (4.20) evolving on the sphere $s^{2}$. Following [3], [9], [10], we define the $\left(\begin{array}{c}p+2 \\ p\end{array}\right)$-vector $x^{[p]}$ consisting of the $p^{\text {th }}$ order moments (homogeneous polynomials) in $\left(x_{1}, x_{2}, x_{3}\right)$ :

$\sqrt{\left(\begin{array}{l}p \\ p_{1}\end{array}\right)\left(\begin{array}{c}p^{-} p_{1} \\ p_{2}\end{array}\right)\left(\begin{array}{c}p-p_{1}-p_{2} \\ p_{3}\end{array}\right)} x_{1}^{p_{1} x_{2}^{p_{2}} x_{3}^{p_{3}} ; \sum_{i=1}^{3} p_{i}=p_{i} \quad p_{i} \geq 0}$

If $y$ satisfies the linear differential equation

$$
\dot{y}(t)=A y(t)
$$

then $y^{[p]}$ satisfies a linear differential equation

$$
\dot{y}^{[p]}(t)=A^{[p]}{ }^{[p]}(t)
$$

We regard this as the definition of $A^{[p]}$. It can be shown that if $x$ satisfies (4.19), then $x^{[p]}$ satisfies

$$
\begin{aligned}
d x^{[p]}(t) & =\left\{\sum_{i=1}^{3} f_{i}(t) A_{i}{ }^{[p]}+\sum_{i, j=1}^{3} Q_{i j}(t) A_{i}{ }^{[p] A_{j}[p]}\right\} x^{[p]}(t) d t \\
& +\sum_{i=1}^{3} A_{i}{ }^{[p]} x^{[p]}(t) d w_{i}(t)
\end{aligned}
$$

The optimal (constrained least-squares) filter is given by the infinite set of coupled equations 


$$
\begin{aligned}
d E^{t}\left[x^{[p]}(t)\right] & =\left\{\sum_{i=1}^{3} f_{i}(t) A_{i}[p]+\sum_{i, j=1}^{3} Q_{i j}(t) A_{i}[p] A_{j}[p]\right\} E^{t}\left[x^{[p]}(t)\right] d t \\
& +\left\{E^{t}\left[x^{[p]}(t) x^{\prime}(t)\right]-E^{t}\left[x^{[p]}(t)\right] E^{t}\left[x^{\prime}(t)\right]\right\} \\
& \cdot H^{\prime}(t) R^{-1}(t)\left[d z(t)-H(t) E^{t}[x(t)] d t\right. \\
\tilde{x}(t) & =\frac{E^{(t)}\left[x^{[1]}(t)\right]}{\left\|E^{t}\left[x^{[1]}(t)\right]\right\| \hat{x}(t \mid t)}\|\hat{x}(t \mid t)\|
\end{aligned}
$$

The structure of this filter is quite similar to that of (7.3) - (7.4) -i.e., it consists of an infinite bank of filters, and the filter for $x^{[p]}$ is coupled only to those for $x$ and $x^{[p+1]}$.

The similarities with the previous example are further illuminated by considering the spherical harmonics [77], [78], which constitute an orthonormal basis for the eigenfunctions of the Laplacian on $s^{2}$. We introduce polar coordinates $(\theta, \phi)$ on $s^{2}$, where $0 \leq \theta \leq \pi, 0 \leq \phi \leq 2 \pi$, by defining

$$
x_{1}=\cos \theta ; x_{2}=\sin \theta \cos \phi ; x_{3}=\sin \theta \sin \phi
$$

The normalized spherical harmonics ${ }^{l_{m}}(\theta, \phi)$ are defined by [77]

$$
\begin{gathered}
\mathrm{Y}_{\ell \mathrm{m}}(\theta, \phi)=(-1)^{\mathrm{m}}\left[\frac{(\ell-\mathrm{m}) !}{(\ell+\mathrm{m}) !} \frac{(2 \ell+1)}{4 \pi}\right]^{1 / 2} \mathrm{P}_{\ell \mathrm{m}}(\cos \theta) \mathrm{e}^{\mathrm{im} \phi} \\
\mathrm{Y}_{\ell,-\mathrm{m}}(\theta, \phi)=(-1)^{\mathrm{m}} \mathrm{Y}_{\ell \mathrm{m}}^{*}(\theta, \phi)
\end{gathered}
$$

for $\ell=0,1, \ldots$ and $m=0,1, \ldots, \ell$, where $\mathrm{P}_{\ell m}(\cos \theta)$ are the associated 
Legendre functions.

According to the remark at the beginning of this section, any square-integrable function on $s^{2}$ can be expanded in a series of the form

$$
f(\theta, \phi)=\sum_{\ell=0}^{\infty} \sum_{m=-\ell}^{\ell} c_{\ell m} Y_{\ell m}(\theta, \phi)
$$

where

$$
c_{\ell m}=\int_{0}^{2 \pi} \int_{0}^{\pi} \mathrm{y}_{\ell m}^{*}(\theta, \phi) f(\theta, \phi) \sin \theta d \theta d \phi
$$

In particular, the conditional probability density function for our estimation problem on $s^{2}$ can be expanded via the series

$$
p(\theta, \phi, t)=\sum_{\ell=0}^{\infty} \sum_{m=-\ell}^{\ell} c_{\ell m}(t) x_{\ell m}(\theta, \phi)
$$

where

$$
C_{\ell_{m}}(t)=E\left[Y_{\ell m}^{*}(\theta(t), \phi(t)) \mid z(s), 0 \leq s \leq t\right]
$$

The set of coefficients $\left\{c_{\ell m}(t)\right\}$ for $\ell=1, \ldots, k$ and $m=-l, \ldots, l$ is equivalent to the set of conditional moments $\left\{E^{t}\left[x^{[p]}(t)\right]\right\}$ for $p=1, \ldots, k$ (see [3] for a detailed description of this equivalence). Thus a filter which generates $\left\{c_{\ell m}(t)\right\}$ is equivalent to $(7.15)$; in fact, it can be shown that such a filter consists of an infinite set of equations (similar in form to (7.15)), and the equation for $C_{L M}$ is coupled only to those for $\left\{c_{\ell m} ; \ell=L-1, L, L+1 ; m=-\ell, \ldots, \ell\right\}$. The optimal estimate is

$$
\tilde{x}^{\prime}(t)=\frac{\left[\sqrt{2} c_{10}(t),-\left(c_{11}(t)+c_{11}^{*}(t)\right), i\left(c_{11}^{*}(t)-c_{11}(t)\right]\right.}{\sqrt{2}\left(c_{10}^{2}(t)+2\left|c_{11}(t)\right|^{2}\right)^{1 / 2}}
$$


This filter is completely analogous to the $s^{1}$ filter $(7.3)-(7.4)$.

We will again employ an "assumed density" approximation in order to obtain a finite-dimensional filter. An $s^{2}$-analog of the folded normal density is obtained as the solution to the standard diffusion equation on $\mathrm{s}^{2}$ :

$$
\frac{\partial p(\theta, \phi, t)}{\partial t}-\frac{1}{2} \beta(t) \nabla^{2} p(\theta, \phi, t)=0
$$

The Green's function for (7.25) is given by the "bilinear series" [79]

$$
G(\theta, \phi, t ; \eta, v, \tau)=\sum_{\ell=0}^{\infty} \sum_{m=-\ell}^{\ell} y_{\ell m}(\theta, \phi) \mathrm{y}_{\ell m}^{*}(\eta, v) e^{-\ell(\ell+1)} \int^{t} \beta(s) d s
$$

This is the solution to (7.25) with initial condition equal to the singular distribution concentrated at $(n, v)$.

Thus by analogy with (7.5), we assume that the conditional density for the $s^{2}$ estimation problem is of the form

$$
\mathrm{p}(\theta, \phi, t)=\sum_{\ell=0}^{\infty} \sum_{m=-\ell}^{\ell} \mathrm{Y}_{\ell \mathrm{m}}(\theta, \phi) \mathrm{Y}_{\ell \mathrm{m}}^{*}(n(t), v(t)) \mathrm{e}^{-\frac{1}{2} \ell(\ell+1) \gamma(t)}
$$

In order words, $C_{\ell m}(t)$ (as defined in (7.22) - (7.23) is assumed to be

$$
c_{\ell m}(t)=Y_{\ell m}^{*}(\eta(t), v(t)) e^{-\frac{1}{2} \ell \cdot(l+1) \gamma(t)}
$$

In this case, if $c_{10}$ and $c_{11}$ have been computed, then

$$
\gamma(t)=\frac{1}{2} \log \left[\frac{4 \pi}{3}\left(c_{10}^{2}(t)+2\left|c_{11}(t)\right|^{2}\right)\right]
$$




$$
\begin{aligned}
& \cos \eta(t)=\frac{c_{10}(t)}{\left[c_{10}^{2}(t)+2\left|c_{11}(t)\right|^{2}\right]^{1 / 2}} \\
& \sin \eta(t)=\frac{\sqrt{2}\left|c_{11}(t)\right|}{\left[c_{10}^{2}(t)+2\left|c_{11}(t)\right|^{2}\right]^{1 / 2}}
\end{aligned}
$$

If $c_{11}(t)=0$, then the density is independent of $v(t)$; otherwise,

$$
e^{2 i v(t)}=\frac{c_{11}^{*}(t)}{c_{11}(t)}
$$

Then $\left\{c_{N+1, m}, m=-(N+1), \ldots, N+1\right\}$ can be computed (for any $N$ ) from

$$
C_{N+1, m}(t)=Y_{N+1, m}^{*}(n(t), v(t)) e^{-\frac{1}{2} \cdot(N+1)(N+2) \gamma(t)}
$$

Thus we can truncate the bank of filters for $\left\{c_{\ell_{m}}\right\}$ by approximating $\left[\mathrm{C}_{\mathrm{N}+1, \mathrm{~m}}\right\}$ via (7.29) $-(7.33)$ and substituting these approximations into the equations for $\left\{\mathrm{c}_{\mathrm{N}, \mathrm{m}}\right\}$.

The performance of this suboptimal filter will be tested by simulation, and the results will be presented in a future report. Other "assumed densities" will also be studied, including those resulting from degenerate diffusions or diffusions with unequal drifts around the three axes [3].

The techniques of this section can in theory be extended to any compact manifold by employing the eigenfunctions of the Laplace-Beltrami operator. For example, on the $n$-sphere $s^{n}$ these functions are the $n-$ dimensional spherical harmonics [78]. Since so(3) is isomorphic (as a Lie 
group) to $s^{3} /\{ \pm I\}$ (see [22], [43]), suboptimal filters for some of the rotational estimation problems of section IV can be constructed by means of spherical harmonics on $\mathrm{s}^{3}$. 
VIII. A Cumulant Method for Suboptimal Filter Design

As we have seen, we are able to obtain finite dimensional optimal filters only for certain bilinear estimation problems. In the preceding section we described how harmonic analysis could be used to design high quality estimation systems for processes evolving on spheres and, more generally, on compact manifolds. In this section we describe a design technique which we call the cumulants method. This approach has been considered by several authors $[10],[59],[60]$ and is related to statistical linearization techniques [60] - [62].

We wish to consider the estimation of $x(t)$ given the observation process $\mathbf{z}$, where these processes satisfy

$$
\begin{aligned}
& d x(t)=a(x(t), t) d t+B(x(t), t) d w(t) \\
& d z(t)=H(t) x(t) d t+R^{1 / 2}(t) d v(t)
\end{aligned}
$$

where $a$ is an $n$-vector and $B$ an $n \times m$ matrix of polynomials in the components of $\mathrm{x}, \mathrm{w}$ and $\mathrm{v}$ are independent standard vector Brownian motion processes, and $R>0$. All of the random processes considered in the preceding sections are of the form. (8.1) (see section II). We consider only the linear observation process (8.2), but the analysis of this section can also be carried out for bilinear and multiplicative processes of the forms given by $(2.12)$ and $(2.13)$.

We wish to compute the conditional moments

$$
m_{k_{1}} \ldots k_{n}(t \mid t)=E\left[x_{1}^{k_{1}}(t) \ldots x_{n}^{k_{n}}(t) \mid z(s), 0 \leq s \leq t\right]
$$

Recall that these quantities were of direct interest in the various estimation problems described earlier. For any twice continuously 
differentiable real-valued function $\phi$ of $x$, let

$$
\hat{\phi}(t \mid t)=E[\phi(x(t)) \mid z(s), 0 \leq s \leq t]
$$

We then have Kushner's stochastic differential equation for $\hat{\phi}[63],[64]$ :

$$
\begin{aligned}
\mathrm{d} \hat{\phi} & =\left\{\widehat{\phi_{\mathbf{x}}}+\frac{1}{2} \operatorname{tr}\left[\widehat{B B^{\prime} \phi}\right] d x d\right. \\
& +\widehat{\phi H x}-\hat{\phi} H \hat{x}] \cdot R^{-1}[d z-H \hat{x}]
\end{aligned}
$$

Note that if $\phi$ is of the form $x_{1}{ }^{k_{1}} \ldots x_{n}{ }_{n}$, the right-hand side of (8.5) consists solely of various conditional moments as in (8.3) (see for example (7.15)). This is a direct consequence of the fact that the right-hand sides of (8.1) and (8.2) contain only polynomial functions of $x$.

A major complication with these equations (when $\hat{\phi}$ is a moment (8.3)) is that they are all coupled together (as in (7.15)). The reason for this is the following: let the order of a moment $m_{k_{1}} \ldots k_{n}$ be the sum of the $k_{i}$. Then, because of the polynomial nature of the various coefficients, the orders of some of the terms on the right-hand side of (8.5) are at least one higher than the order of $\phi$. Consider the scalar example

$$
\begin{aligned}
& d x(t)=\alpha x^{2}(t) d t+\beta x(t) d w(t) \\
& d z(t)=x(t) d t+d v(t)
\end{aligned}
$$

Then

$$
\begin{aligned}
& d m_{1}=\alpha m_{2} d t+\left[m_{2}-m_{1}^{2}\right]\left[d z-m_{1} d t\right] \\
& d m_{2}=\left[2 \alpha m_{3}+\beta^{2} m_{2}\right] d t+\left[m_{3}-m_{2} m_{1}\right]\left[d z-m_{1} d t\right]
\end{aligned}
$$




$$
d m_{3}=\left[3 \alpha m_{4}+3 \beta^{2} m_{3}\right] d t+\left[m_{4}-m_{3} m_{1}\right]\left[d z-m_{1} d t\right]
$$

From the preceding comments, it is clear that the implementation of these equations must involve an approximation -- i.e., a truncation of the infinite set of equations. For several reasons, the direct truncation method -- setting to zero all moments greater than some given order -- can cause difficulties. First of all, there is no reason to expect the higher moments to be small, and in many cases (such as the Gaussian case) the sequence of moments is unbounded. In addition, if we use the fact that the moments are the coefficients of the Taylor series expansion of the characteristic function of $x$, the assumption that the higher moments are zero corresponds to assuming that the density for $x$ is a sum of derivatives of Dirac delta functions [59].

As suggested in [59] and [60], a more useful set of variables is the set of cumulants, which are the coefficients of the Taylor series expansion of the logarithm of the characteristic function. For the present discussion we limit ourselves to the scalar case, although the vector case can be handled similarly. For a scalar variable the kth cumulant is a polynomial in the first $k$ moments and, in fact, the first $k$ moments and the first $k$ cumulants contain precisely the same information. For example, the first 4 cumulants of the scalar process $x(t)$ in $(8.6)$ are

$$
\begin{aligned}
& k_{1}(t)=m_{1}(t) \\
& k_{2}(t)=m_{2}(t)-m_{1}^{2}(t) \\
& k_{3}(t)=m_{3}(t)-3 m_{1}(t) m_{2}(t)+2 m_{1}^{3}(t)
\end{aligned}
$$




$$
k_{4}(t)=m_{4}(t)-3 m_{2}^{2}(t)-4 m_{1}(t) m_{3}(t)+12 m_{1}^{2}(t) m_{2}(t)-6 m_{1}^{4}(t)
$$

and we see that $k_{1}$ is the mean and $k_{2}$ is the variance.

As discussed in [59], a reasonable procedure is to set to zero

all cumulants of order higher than some given number. Note that

assuming $k_{i}=0 \forall i \geq 3$ is equivalent to assuming that $x(t)$ is Gaussian! Note also that if we take $k(t)=0, i \geq M$, we can obtain equations for the corresponding higher moments of $x(t)$. In this way, we can effectively truncate the infinite set of moment equations. For instance, if we return to the scalar example (8.6), (8.7), and if we assume $k_{i}(t)=0 \forall i \geq 4$ equations (8.8) and (8.9) are unchanged and (8.10) is replaced by

$$
\begin{aligned}
& d m_{3}=\left[3 a \eta\left(m_{1}, m_{2}, m_{3}\right)+3 \beta^{2} m_{3}\right] d t \\
&+\left[\eta\left(m_{1}, m_{2}, m_{3}\right)-m_{3} m_{1}\right]\left[d z-m_{1} d t\right] \\
& n\left(m_{1}, m_{2}, m_{3}\right)=3 m_{2}^{2}+4 m_{1} m_{3}-12 m_{1}^{2} m_{2}+6 m_{1}^{4}
\end{aligned}
$$

We note that this technique can be extended to the general vector problem (8.1), (8.2) with no conceptual, but some bookkeeping, difficulties. For instance, the cumulants approach provides an alternative to the Fourier series methods described in section VII for the design of phase tracking and demodulation systems. An open question related to the cumulants method is that of performance analysis -- e.g., how does performance improve as we retain more cumulants and do we achieve the optimal performance in the limit? 


\section{Conclusions}

In this paper we have discussed the practical importance and the mathematical analysis of several classes of bilinear estimation problems. We have seen that such mathematical models arise in a wide variety of applications, and we refer the reader to the references for further verification of this fact. We have also indicated how such estimation problems can be solved. In some cases, best explained in a Lie-theoretic framework, we have seen that finite-dimensional optimal estimation equations can be derived. For other problems the tools of harmonic analysis have turned out to be most appropriate and extremely useful. Finally, a general but untried approximation method based on the truncation of the cumulants of a random process has been described. In conclusion, the class of stochastic bilinear systems is not only an appealing class of systems from an applications point of view, but it also is a highly structured class of systems for which analysis nearly as detailed and successful as that for linear systems. is possible. 


\section{$\underline{\text { References }}$}

1. R.W. Brockett, "System Theory on Groups Manifolds and Coset Spaces," SIAM J. Control, Vol. 10, No. 2, May 1972, pp. 265-284.

2. R.w. Brockett, "On the Algebraic Structure of Bilinear systems," in Theory and Applications of Variable structure Systems, R. Mohler and A. Ruberti, eds., Academic Press, New York, 1972 .

3. R.W. Brockett, "Lie Algebras and Lie Groups in Control Theory," in Geometric Methods in System Theory, D.Q. Mayne and R.W. Brockett, eds., Reidel Pub. Co., The Netherlands, 1973.

4. v. Jurdjevic and H.J. Sussmann, "Control Systems on Lie Groups," J. Diff. Egns., Vol. 12, No. 2, 1972, pp. 313-329.

5. R.M. Hirschorn, "Controllability in Nonlinear Systems," in Geometric Methods in System Theory, D.Q. Mayne and R.W. Brockett, Eds., Reidel Pub. Co., The Netherlands, 1973.

6. A. Isidori and A. Ruberti, "Realization Theory of Bilinear Systems," in Geometric Methods in System Theory, D.Q. Mayne and R.W. Brockett, eds., Reidel Pub. Co., The Netherlands, 1973.

7. R. Mohler, "Bilinear Structures and Man," in Theory and Applications of Variable Structure Systems, R. Mohler and A. Ruberti, eds., Academic Press, New York, 1972.

8. C. Bruni, G. DiPillo, and G. Koch, Bilinear Systems: An Appealing Class of "Nearly Linear" Systems in Theory and Applications, Universita'di Roma, Istituto di Automatica, R. 2-29, December, 1972

9. R.W. Brockett, "Lie Theory and Control Systems on Spheres," SIAM J. Appl. Math., Vol. 25, No. 2, Sept. 1973, pp. 213-225.

10. A.S. Willsky and S.I. Marcus, "Analysis of Bilinear Noise Models in Circuits and Devices," Monograph of the Colloguium on the Application of Lie Group Theory to Nonlinear Network Problems, 1974 IEEE International Symposium on Circuits and Systems, San Francisco, Calif., April 1974.

11. G.L. Blankenship, "Perturbation Theory for Stochastic Ordinary Differential Equations with Applications to Optical Waveguide Analysis," Monograph of the Colloguium on the Application of Lie Groups Theory to Nonlinear Network Problems 1974 IEEE International Symposium on Circuits and systems, San Francisco, Calif., April 1974.

12. H.P. McKean, Jr., Stochastic Integrals, Academic Press, N.Y., 1969. 
13. H.P. McKean, Jr., "Brownian Motion on the 3-Dimensional Rotation Groups," Mem. Coll. Sci. Kyoto Univ., Vol. 33, 1960, pp. 25-38.

14. J.T. Lo, "Bilinear Stochastic Systems and Finite Dimensional Sensor Orbits," Eighth Princeton Conference on Information Sciences and Systems, Princeton, New Jersey, March 28-29, 1974.

15. G. Koch, "Some Results on Stochastic Bilinear Systems," Fourth Symposium on Nonlinear Estimation and Its Applications," San Diego, Calif., Sept. 10-12, 1973.

16. A.S. Willsky, Dynamical Systems Defined on Groups: Structural Properties and Estimation, Ph.D. Thesis, Dept. of Aeronautics and Astronautics, M.I.T., Cambridge, Mass., June 1973.

17. J.T. Lo and A.S. Willsky, "Estimation for Rotational Processes with One Degree of Freedom I: Introduction and Continuous Time Processes," IEEE Trans. on Aut. Cont., to appear.

18. A.S. Willsky and J.T. Lo, "Estimation for Rotational Processes with One Degree of Freedom II: Discrete Time Processes," IEEE Trans. on Aut. Control, to appear.

19. A.S. Willsky and J.T. Lo, "Estimation for Rotational Processes with One Degree of Freedom III: Applications and Implementation," IEEE Trans. on Aut. Control, to appear.

20. A.S. Willsky, "Estimation and Detection of Signals in Multiplicative Noise," submitted to IEEE Trans. on Aut. Control.

21. A.S. Willsky, "Some Estimation Problems on Lie Groups," in Geometric Methods in System Theory, R.W. Brockett and D.Q. Mayne, eds., Reidel Pub. Co., The Netherlands, 1973.

22. A.S. Willsky, "Some Results on the Estimation of the Angular Velocity and Orientation of a Rigid Body," submitted to Automatica.

23. J.T. Lo and A.S. Willsky, "Stochastic Control of Rotational Processes with One Degree of Freedom," submitted to SIAM J. Control.

24. A.S. Willsky, "Fourier Series and Estimation on the Circle with Applications to Synchronous Communication I: Analysis," IEEE Trans. on Inf. Theory, to appear.

25. A.S. Willsky, "Fourier Series and Estimation on the Circle with Applications to Synchronous Communication II: Implementation," IEEE Trans. on Inf. Theory, to appear.

26. J.T. Lo, "Signal Detection on Lie Groups," IEEE Trans. on Inf. Theory, to appear. 
27. B.W. Stuck, Space Satellite Dynamics with Applications to Sunlight Pressure Attitude Control, Ph.D. Thesis, Dept. of Electrical Engineering, M.I.T., Cambridge, Mass., June 1972.

28. A.H. Jazwinski, Stochastic Processes and Filtering Theory, Academic Press, New York, 1970.

29. E. Wong, Stochastic Processes in Information and Dynamical Systems, McGraw-Hil1 Book Co., New York, 1971.

30. E.J. McShane, "Toward a Stochastic Calculus, I," Proc. N.A.S., Vol. 63,1969 , pp. 275-280.

31. E.J. MaShane, "Toward a Stochastic Calculus, II," Proc. N.A.S., Vol. $63,1969,1084-1087$.

32. H.L. Van Trees, Detection, Estimation, and Modulation Theory, Part II Nonlinear Modulation Theory, John Wiley and Sons, Inc., New York, $\overline{1971}$

33. A.J. Viterbi, Principles of Coherent Communication, McGraw-Hill Book Co., New York, 1966.

34. D.E. Gustafson and J.L. Speyer, "Linear and Asymptotic Minimum Variance Filters Applied to Phase-Lock Loops," IEEE Trans. on Comm., to appear.

35. R.S. Bucy, C. Hecht, and K.D. Senne, "New Methods for Nonlinear Filtering," Revue Francaise d'Automatique, Informatique et de Recherche Operationnelle, Feb. 1973, pp. 3-54.

36. J.J. Mallinckrodt, R.S. Bucy, and S.Y. Chang, Final Project Report for a Design study for an Optimal Non-Linear Receiver/Demodulator, NASA Contract NAS 5-10789, Goddard Space Flight Center, Maryland, 1970.

37. J.M. Wozencraft and I.M. Jacobs, Principles of Communication Engineering, John Wiley and Sons, Inc., New York, 1965.

38. J.A. Pierce, W. Palmer, A.D. Watt, and R.H. Woodward, Omega -- A World-Wide Navigational system Specification and Implementation -Second Revision, Pickard and Burns Electronics, Waltham, Mass., May 1966.

39. M.G. Crosby, "Frequency-Modulation Noise Characteristics," Proc. of the IRE, Vol. 25, April 1937, pp. 472-514.

40. L.S. Cutler and C.L. Searle, "Some Aspects of the Theory and Measurement of Frequency Fluctuations in Frequency standards, Proc. of the IEEE, Vol. 54, No. 2, Feb. 1966, pp. 136-154. 
41. M. Kayton and W.R. Fried, eds., Avionics Navigation Systems, John Wiley and Sons, Inc., New. York, 1969.

42. C. Chevalley, Theory of Lie Groups, Princeton University Press, Princeton, $194 \overline{6}$.

43. F. Warner, Foundations of Differential Manifolds and Lie Groups, Scott, Foresman and Co., Glenview, Ill., 1971.

44. E.V. Hoversten, R.O. Harger, and S.J. Halme, "Communication Theory for the Turbulent Atmosphere," Proc. of the IEEE, Vol. 58, No. 10, Oct. 1970, pp. 1626-1650.

45. R.L. Halfman, Dynamics Vol. I, Addison-Wesley Pub. Co., Inc., Reading, Mass., 1962.

46. w. Wrigley, w. Hollister, and w. Denhard, Gyroscopic Theory, Design, and Instrumentation, The M.I.T. Press, Cambridge, Mass., 1969.

47. A.E. Bryson, Jr. and W. Kortum, "Estimation of the Local Attitude of Orbiting Spacecraft," Automatica, Vol. 7, 1971, pp. 163-180.

48. B.W. Stuck, "A New Method for Attitude Estimation," presented at the AAS/AIAA Astrodynamics Conference, Vail, Colorado, July 16-18, 1973.

49. R.W. Brockett and H.J. Sussmann, "Tangent Bundles of Homogeneous Spaces are Homogeneous Spaces," Proc. Amer. Math. Soc., Vol. 35, No. 2, Oct. 1972, pp. 550-551.

50. H. Samelson, Notes on Lie Algebras, Van Nostrand Reinhold Co., New York, 1969.

51. J. Wei and E. Norman, "Lie Algebraic Solution of Linear Differential Equations," J. Mathematical Physics, Vol. 4, 1963, pp. 575-581.

52. J. Wei and E. Norman, "On Global Representations of the Solutions of Linear Differential Equations as a Product of Exponentials," Proc. Amer. Math. Soc., Vol. 15, 1964, pp. 327-334.

53. A.E. Bryson, Jr. and D.E. Johansen, "Linear Filtering for TimeVarying Systems Using Measurements Containing Colored Noise," IEEE Trans. Aut. Control, Vol. AC-10, 1965, pp. 4-10.

54. J.T. Lo, "Signal Detection of Rotational Processes and Frequency Demodulation," Proc. of the 1973 IEEE Conference on Decision and Control, San Diego, December 1973.

55. R.W. Brockett and J.R. Wood, "Electrical Networks Containing Controlled Switches," Monograph of the Colloguium on the Application of Lie Group Theory to Nonlinear Network Problems, 1974 IEEE International Symposium on Circuits and Systems, San Francisco, Calif., April 1974. 
56. T.G. Dankel, Jr., "Mechanics on Manifolds and the Incorporation of Spin into Nelson's Stochastic Mechanics," Arch. Rat. Mech. Anal., vol. 37, No. 3, 1970, pp. 192-221.

57. J.E. Potter and E.J. Frey, Rotation Invariant Probability Distributions on the Surface of a Sphere, with Applications to Geodosy, M.I.T. Experimental Astronomy Lab. Report No. RE-27, May 1967.

58. B.D. Tapley and D.S. Ingram, "Orbit Determination in the Presence of Unmodeled Accelerations," IEEE Trans. on Aut. Control, Vol. AC-18, August 1973, pp. 369-373.

59. R.L. Stratonovich, Topics in the Theory of Random Noise, Volume I, Translated by R.A. Silverman, Gordon and Breach, New York, 1963.

60. T. Nakamizo, "On the State Estimation for Non-Linear Dynamic Systems," Int. J. Control, Vol. 11, No. 4, 1970, pp. 683-695.

61. R.J. Phaneuf, Approximate Nonlinear Estimation, Ph.D. Thesis, Dept. of Aeronautics and Astronautics, M.I.T., Cambridge, Mass., May 1968.

62. Y. Sunahara, Technical Report 67-8, Center for Dynamical Systems, Div. of Applied Math., Brown Univ., Providence, R.I., 1967.

63. A.A. Desalu, Stochastic Modeling and Dynamic Estimation of Air Quality in an Advective-Diffusive Atmosphere, Ph.D. Thesis, Dept. of Electrical Engineering, M.I.T., Cambridge, Mass., 1974.

64. A.A. Desalu, L.A. Gould, and F.C. Schweppe, "Dynamic Estimation of Air Pollution," submitted to IEEE Trans. on Aut. Control.

65. T.J. Tarn, D.L. Elliott, and T. Goka, "Controllability of Discrete Bilinear Systems with Bounded Control," IEEE Trans. on Aut. Control, Vol. AC-18, 1973, pp. 298-301.

66. A. Isidori, "Direct Construction of Minimal Bilinear Realizations from Nonlinear Input-Output Maps," IEEE Trans. on Aut. Control, Vol. AC-18, 1973, pp. 626-631.

67. T. Kailath, "Some Chandrasekar-Type Algorithms for Linear Quadratic Regulators," Proc. of the 1972 IEEE Conf. on Decision and Control, New Orleans, December 1972.

68. A. Lindquist, "A New Algorithm for Optimal Filtering of Discrete-Time Stationary Processes," SIAM J. Control, to appear.

69. A.A. Sagle and R.E. Walde, Introduction to Lie Groups and Lie Algebras, New York, Academic Press, 1973. 
70. R.W. Brockett, Finite Dimensional Linear Systems, New York, John Wiley, 1970.

71. C.T. Leondes, J.B. Peller, and E.B. Stear, "Nonlinear Smoothing Theory," IEEE Trans. Syst. Sci. Cyber., Vol. SSC-6, 1970, p.63.

72. H. Kwakernaak, "Optimal Filtering in Linear Systems with Time Delays," IEEE Trans. on Aut. Control, Vol. AC-12, 1967, pp. 169-173.

73. T. Kailath and P. Frost," An Innovations Approach to Least-Squares Estimation, Part II: Linear Smoothing in Additive White Noise," IEEE Trans. on Aut. Control, Vol. AC-13, 1968, pp. 655-660.

74. W. Rudin, Real and Complex Analysis, New York, McGraw-Hill, 1966.

75. K.S. Miller, Multidimensional Gaussian Distributions, New York, John Wiley, 1965 .

76. L.H. Loomis, An Introduction to Abstract Harmonic Analysis, Princeton, Van Nostrand, 1953.

77. J.D. Talman, Special Functions -- A Group Theoretic Approach, New York, Benjamin, 1968.

78. A. Erdelyi, W. Magnus, F.Oberhettinger, and F. Tricomi, Higher Transcendental Functions, Vol. II, New York, McGraw-Hill, 1953.

79. R. Dennemeyer, Introduction to Partial Differential Equations and Boundary Value Problems, New York, McGraw-Hill, 1968.

80. W. Kohn, T.L. Johnson, and A.S. Willsky, "Analysis of a Mathematical Model for Transport Phenomena," to appear. 